\title{
Finite-SNR analysis for partial relaying cooperation with channel coding and opportunistic relay selection
}

\author{
Thang X. Vu ${ }^{1 *} \mathbb{D}$, Pierre Duhamel ${ }^{2}$, Symeon Chatzinotas ${ }^{1}$ and Bjorn Ottersten ${ }^{1}$
}

\begin{abstract}
This work studies the performance of a cooperative network which consists of two channel-coded sources, multiple relays, and one destination. To achieve high spectral efficiency, we assume that a single time slot is dedicated to relaying. Conventional network-coded-based cooperation (NCC) selects the best relay which uses network coding to serve the two sources simultaneously. The bit error rate (BER) performance of NCC with channel coding, however, is still unknown. In this paper, we firstly study the BER of NCC via a closed-form expression and analytically show that NCC only achieves diversity of order two regardless of the number of available relays and the channel code. Secondly, we propose a novel partial relaying-based cooperation (PARC) scheme to improve the system diversity in the finite signal-to-noise ratio (SNR) regime. In particular, closed-form expressions for the system BER and diversity order of PARC are derived as a function of the operating SNR value and the minimum distance of the channel code. We analytically show that the proposed PARC achieves full (instantaneous) diversity order in the finite SNR regime, given that an appropriate channel code is used. Finally, numerical results verify our analysis and demonstrate a large SNR gain of PARC over NCC in the SNR region of interest.
\end{abstract}

Keywords: Cooperative diversity, Relay selection, Partial relaying, Channel coding

\section{Introduction}

Cooperation among nodes is an effective technique to widen the coverage and to improve the performance of wireless networks both in terms of signal-to-noise ratio (SNR) and diversity gain $[1,2]$. Such improvements, however, usually comes at the price of an additional orthogonal channel, resulting in a reduced spectral efficiency, which can be significant in multiple-relay networks. In order to reduce this loss, opportunistic relay selection (RS) has been proposed to select the best relay for cooperation [3]. It has been shown that RS achieves full diversity order for single-source multiple-relay networks and outperforms other relaying schemes in terms of SNR gain and effective capacity [4].

Network coding (NC) has gained tremendous attention because of its potential improvement in diversity gain

\footnotetext{
*Correspondence: thang.vu@uni.lu

${ }^{1}$ Interdisciplinary Centre for Security, Reliability and Trust (SnT), University of Luxembourg, Luxembourg, Luxembourg
}

Full list of author information is available at the end of the article and throughput over classical routing techniques [5]. The principle of $\mathrm{NC}$ is to allow intermediate nodes to combine multiple input packets into a single output. Recently, there has been much interest on combining $\mathrm{NC}$ together with RS to further improve the spectral efficiency. It is shown via outage probability (OP) analysis that the use of RS in a two-way relay channel (TWRC) could achieve full diversity order and a significant SNR gain [6-9]. The authors in [6] propose a joint design of NC with RS for decode-and-forward (DF) TWRC based on the max-min criterion in order to maximize the worst relay channel. In [7], an SNR-based suboptimal relay ordering is proposed for two-way amplify-and-forward (AF) relay networks. A similar method is studied in [8] to derive the BER, OP, and diversity order. While research on RS in TWRC is readily available in the literature, research on RS in unidirectional relay networks is still limited. This problem was first considered in [10], which analyzes diversity multiplexing tradeoff (DMT) and shows that full diversity 
order is achieved. However, [10] relies on an unrealistic assumption that unintended packets are available at all destinations. A generalized DMT analysis is presented in [11]. Likewise, [11] relies on an optimistic assumption that the selected channels are independent, which is infeasible because these channels belong to an ordered SNR sequence, and thus are highly correlated [7]. By removing this unrealistic assumption, it was shown in our previous work that NCC fails to achieve full underlying diversity gain [12] regardless of the number of available relays. The analysis of the counterpart AF in inter-user interference channels was studied in [13-16]. In [17, 18], the impacts of outdated and imperfect channel state information (CSI) were analyzed via closed-form expression for system $O P$ and pair-wise error probability. It is worth noting that the abovementioned works study the system diversity via the upper-bound limit of the BER or OP in the absence of channel coding, which is not the case in many practical scenarios where nodes are usually protected by some forward error correction codes.

In this paper, we investigate the performance of cooperative networks under practical conditions, i.e., the transmitted signals are protected by convolutional codes (CC). In the considered system, two sources communicate with a common destination with the aid of multiple relays. Such a scenario can find applications in the uplink cellular mobile systems where two mobile users send data to the base station and some friendly, idle users act as relays. Due to the constraints on spectral efficiency and processing delay, it is assumed that only one time slot is dedicated to cooperation. The best RS is employed to effectively exploit the spatial diversity [3]. At the destination, cooperative maximal ratio combining (C-MRC) detector [19] is employed prior to channel decoding to mitigate error propagation. We note that C-MRC is a suboptimal detector but provides full diversity gain and performance close to the maximum likelihood (ML) receiver [20].

Our contributions are as follows:

- Firstly, we analyze the BER in closed-form expressions for the NCC, in which one selected relay helps the two sources by applying network coding on the estimated codewords. From the analyzed BER, we analytically show that NCC always achieves a diversity of order two regardless of the channel code and the total number of relays. This result coincides with the diversity order derived from OP analysis in $[11,12]$. It would be noted that we analyze NCC in the presence of RS and channel coding, whereas [21] considered a single-relay network and [9] studied symbol-based NCC without channel coding.

- Secondly, we propose a partial relaying based cooperation (PARC). The key difference between PARC and NCC is that the former selects two relays for cooperation, each one helping one source independently. Compared to [22, 23], our proposed scheme has two main differences: (i) we analyze the system via BER, whereas these papers study the system OP, which is fundamentally different from our setting (we can obtain the actual BER for arbitrary SNR value); and (ii) we investigate RS to improve the spectral efficiency, while these papers consider single-relay networks.

- Thirdly, insightful theoretical analysis is provided for PARC in the finite-SNR regime. In particular, closed-form expressions for the BER and instantaneous diversity order ${ }^{1}$ are derived, which reveal the dependency of the instantaneous diversity order on the operating SNR value and the minimum distance of the channel code.

- Finally, numerical results demonstrate the effectiveness of our proposed scheme. It is shown via both analytical and simulation results that PARC can achieve full (instantaneous) diversity order in the low and medium SNR regime when a suitable CC is used. This result is important since the practical systems usually operate in the finite SNR regime.

The rest of the paper is organized as follows: Section 2 describes the system model. Section 3 provides details for the relay selection process. Section 4 analyzes the BER and diversity order of NCC. Section 5 analyses the performance analysis of PARC. Section 6 shows numerical results. Finally, Conclusions and Discussions are given in Section 7.

\section{System model}

The system under consideration consists of two sources denoted by $\mathrm{S}_{1}$ and $\mathrm{S}_{2}, N_{r}$ relays denoted by $R_{i}$ with $1 \leq$ $i \leq N_{r}$, and one destination denoted by $D$, as depicted in Fig. 1. This scenario can find applications in cellular uplinks in which some idle users help two mobile users to communicate with the base station. All nodes are equipped with a single antenna and operate in half-duplex

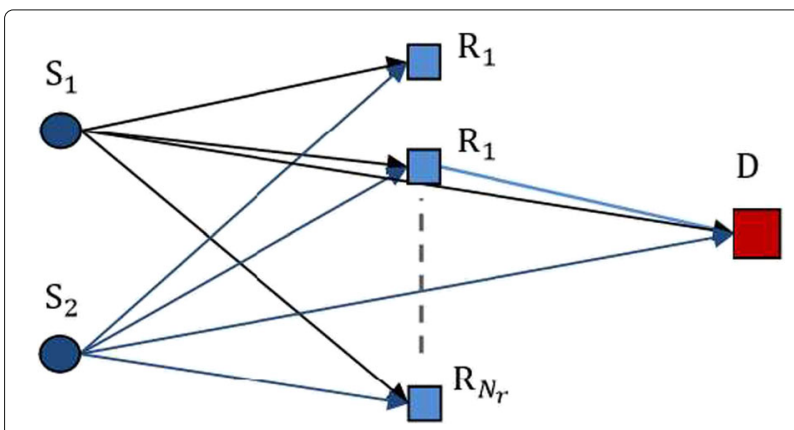

Fig. 1 Considered system consists of two sources: multiple relays and one destination 
mode. We assume orthogonal block Rayleigh fading channels and perfect time synchronization. As a result, one cooperation period is divided into two phases: broadcast and relaying. In the first phase, the two sources consecutively broadcast data to the relays and destination. In the second phase, the selected relay forwards signal to the destination. Demodulate-and-forward relaying protocol [24] is used to minimize the relay's computational complexity. Due to the spectral efficiency constraint and processing time limit, we assume that a single time slot is dedicated to the relaying phase. Furthermore, to effectively exploit spatial diversity and to achieve high spectral efficiency, the best RS is employed [3]. The relay selection process is performed at the beginning of every cooperation period and is described in details in Section 3.

\subsection{Network coding-based cooperation (NCC)}

In NCC, the relays use network coding to help both sources simultaneously to improve the spectral efficiency. Only one best relay is selected to forward the whole network-coded codeword to the destination. The time allocation of NCC is depicted in Fig. 2a.

First, source $S_{i}$ with $i=1,2$ encodes a $K$-length data message $\mathbf{u}_{i}$ into a codeword $\mathbf{c}_{i}$ by a convolutional code $\mathbf{g}$ with code rate $K / N$. Each codeword $\mathbf{c}_{i}$ contains $N$ coded symbols. The codeword $\mathbf{c}_{i}$ is then modulated into a signal $\mathbf{x}_{i}$. Next, the signal $\mathbf{x}_{i}$ is broadcasted to the relays and the destination. Without loss of generality, denote $\mathrm{R}_{N C}$ as the selected relay in NCC. The received signal at $\mathrm{R}_{N C}$ and the destination are given as

$$
\begin{aligned}
\mathbf{y}_{S_{i} R_{N C}} & =\sqrt{P_{S_{i} R_{N C}}} h_{S_{i} R_{N C}} \mathbf{x}_{i}+\mathbf{n}_{S_{i} R_{N C}}, i=1,2, \\
\mathbf{y}_{S_{i} D} & =\sqrt{P_{S_{i} D} D} h_{S_{i} D} \mathbf{x}_{i}+\mathbf{n}_{S_{i} D}, i=1,2,
\end{aligned}
$$

where $P_{X Y}$ with $X \in\left\{S_{1}, S_{2}\right\}, Y \in\left\{R_{N C}, D\right\}$ is the average received power at node $Y$ from node $X$, including the path loss; $h_{X Y}$ is the channel fading coefficient between $X$ and
$Y$, which is independent and identically distributed (i.i.d.) complex Gaussian random variable with zero mean and unit variance, i.e., $\mathbb{E}\left\{\left|h_{X Y}\right|^{2}\right\}=1$, and is mutually independent among $X \rightarrow Y$ channels; $\mathbf{n}_{(.)}$is a noise vector whose components are i.i.d. complex Gaussian random variables with zero mean and variance $\sigma^{2}$.

At the end of the first phase, $\mathrm{R}_{N C}$ decodes the estimate $\hat{\mathbf{x}}_{i R}$ of $\mathbf{x}_{i}, i=1,2$, using the ML detector as $\hat{c}_{i R, k}=\arg \min _{c_{i, k} \in\{0,1\}}\left\{\left|y_{S_{i} R_{N C}, k}-\sqrt{P_{S_{i} R_{N C}}} h_{S_{i} R_{N C}} x_{i, k}\right|^{2}\right\}, i \in$ $\{1,2\}, 1 \leq k \leq N$, where $x_{i, k}$, the $k$-th symbol of $\mathbf{x}_{i}$, is the modulated symbol of $c_{i, k}$. Then $\mathrm{R}_{N C}$ performs network encoding to get $\hat{\mathbf{c}}_{N C}$, where $\hat{c}_{N C, k}=\hat{c}_{1 R, k} \oplus \hat{c}_{2 R, k}, \forall k$, and $\oplus$ denote the binary XOR operation.

The received signal at the destination from the selected relay is given as

$$
\mathbf{y}_{R_{N C} D}=\sqrt{P_{R_{N C} D}} h_{R_{N C} D} \hat{\mathbf{x}}_{N C}+\mathbf{n}_{R_{N C} D},
$$

where $\hat{\mathbf{x}}_{N C}$ is the modulated signal of $\hat{\mathbf{c}}_{N C}$. After two phases, the destination receives three channel observations from two sources and the selected relay. To decode the source data, the destination applies the joint network/channel decoding algorithm to a "compound code" G [21] which sees the relayed signal as additional parity bits (redundancy). The compound code $\mathbf{G}$ is formed from the individual code $\mathbf{g}$ as follows:

$$
\mathbf{G}=\left[\begin{array}{lll}
\mathbf{g} & \mathbf{0} & \mathbf{g} \\
\mathbf{0} & \mathbf{g} & \mathbf{g}
\end{array}\right]
$$

where 0 is a zero matrix with the same size as g. See [21] for full details of the joint decoding at the destination.

\subsection{Partial relaying-based cooperation (PARC)}

Motivated by our previous work which shows that full diversity order can be achieved for the three-node relay network in the low and medium SNR regimes even when parts of the codeword is forwarded [21], we propose to

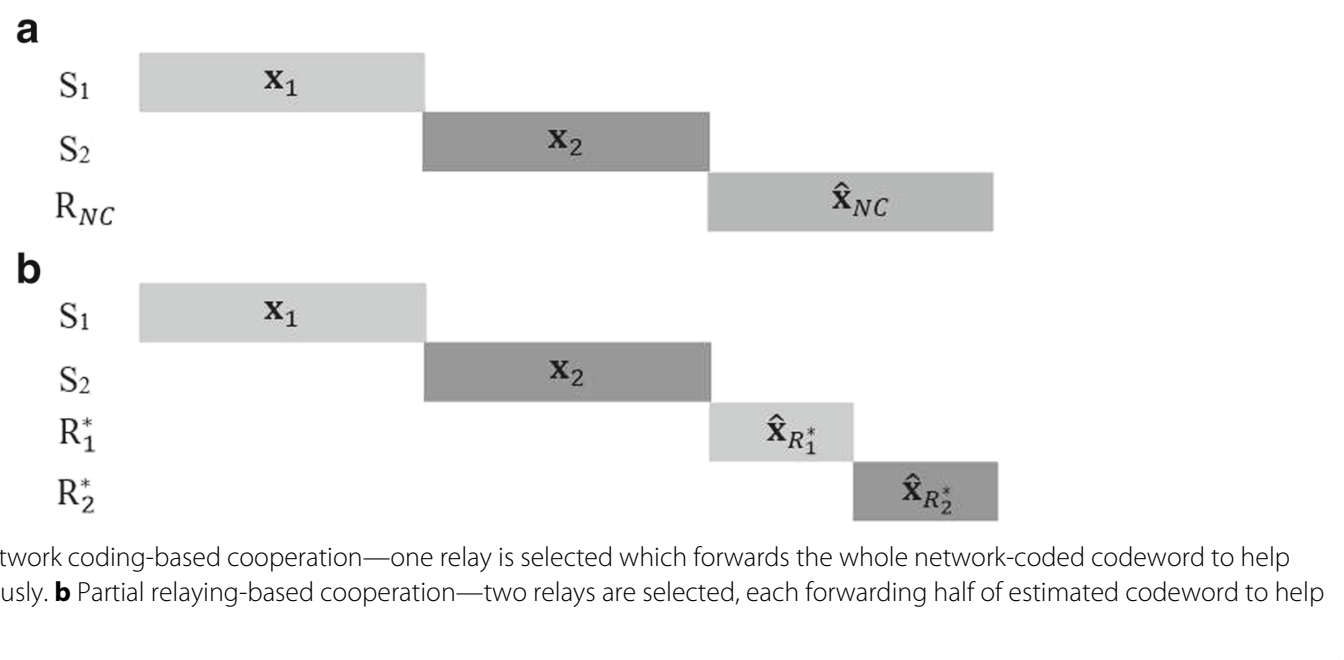

Fig. 2 Time allocation. a Network coding-based cooperation-one relay is selected which forwards the whole network-coded codeword to help the two sources simultaneously. b Partial relaying-based cooperation-two relays are selected, each forwarding half of estimated codeword to help one source independently 
combine RS with partial relaying in PARC to select two relays, each one is the best relay (maximizing the end-toend SNR) for one source. Since there are two active relays in the relaying phase, each relay only occupies half of the relaying time slot, as shown in Fig. 2b. Consequently, the selected relays can only forward half of the estimated codeword to the destination.

Denote $\mathrm{R}_{i}^{\star}, i=1,2$, as the selected relay for $\mathrm{S}_{\mathrm{i}}$ in PARC. The received signals at the selected relays at the end of first phase are given as

$$
\mathbf{y}_{S_{i} R_{i}^{\star}}=\sqrt{P_{S_{i} R_{i}^{*}}} h_{S_{i} R_{i}^{\star}} \mathbf{x}_{i}+\mathbf{n}_{S_{i} R_{i}^{\star}}, i=1,2 .
$$

At the end of the first phase, the selected relay estimates the source coded symbols and forwards them to the destination. In the proposed scheme, the selected relay $\mathrm{R}_{i}^{\star}, i=$ 1,2 , uses half of the relaying time slot to forward half of the codeword $\mathbf{c}_{i}$ to the destination. More specifically, the selected relay $\mathrm{R}_{i}^{\star}$ first estimates $L=N / 2$ (without loss of generality, assuming $N$ is even) source coded symbols to form an estimated punctured codeword $\hat{\mathbf{c}}_{R_{i}^{\star}}=\left\{\hat{c}_{R_{i}^{\star}, l}\right\}_{l \in \Theta}$, where

$\Theta=\left\{k_{1}, k_{2}, \ldots, k_{L}\right\}$ being the set of the indexes of the symbols which are helped by $R_{i}^{\star}$. The index set $\Theta$ is determined randomly ${ }^{2}$. The source coded symbols at the relay are estimated by the ML detector as follows:

$$
\hat{c}_{R_{i}^{\star}, l}=\arg \min _{c_{i, k_{l}} \in\{0,1\}}\left\{\left|y_{S_{i} R_{i}^{\star}, k_{l}}-\sqrt{P_{S_{i} R_{i}^{\star}}} h_{S_{i} R_{i}^{\star}} x_{i, k_{l}}\right|^{2}\right\},
$$

$\forall k_{l} \in \Theta$, where $x_{i, k_{l}}$ being the corresponding modulated symbol of $c_{i, k_{l}}$. Next, $\mathrm{R}_{i}^{\star}$ modulates $\hat{\mathbf{c}}_{R_{i}^{\star}}$ into the modulated signal $\hat{\mathbf{x}}_{R_{i}^{\star}}$ and then forwards it along with the index set $\Theta$ to the destination. The cost for conveying the index set is negligible since it can send, e.g., the seed of the random interleaver, to the destination.

The received signal at the destination transmitted from the relay is given as:

$$
\mathbf{y}_{R_{i}^{\star} D}=\sqrt{P_{R_{i}^{\star} D}} h_{R_{i}^{\star} D} \hat{\mathbf{x}}_{R_{i}^{\star}}+\mathbf{n}_{R_{i}^{\star} D}, i=1,2,
$$

where $h_{R_{i}^{\star} D}$ is the channel coefficient from $\mathrm{R}_{i}^{\star} \rightarrow \mathrm{D}$, and $\mathbf{n}_{R_{i}^{\star} D}$ is a noise vector whose components are Gaussian random variable with zero mean and variance $\sigma^{2}$.

After receiving two signals from $S_{i}$ (see (2)) and $\mathrm{R}_{i}^{\star}$, the destination starts the decoding process with two consecutive steps: demodulating and decoding. We assume that the destination has full CSI knowledge of its incoming channels, i.e., $\mathrm{S}_{i} \rightarrow \mathrm{D}$ and $\mathrm{R}_{i}^{\star} \rightarrow \mathrm{D}$, and of source-toselected relay channels, i.e., $S_{i} \rightarrow R_{i}^{\star}$. These CSIs can be effectively obtained during the pilot transmission. In order to inform the destination of the $\mathrm{R}_{i}^{\star} \rightarrow$ D CSI, the selected relay $\mathrm{R}_{i}^{\star}$ forwards the quantized version of this CSI to the destination. It has been shown in [25] that with 6bit quantization, the destination can achieve similar BER and diversity as the full CSI case in the finite-SNR regime.
Therefore, the overhead for sending these CSIs is negligible. The destination first applies the C-MRC detector [19] to demodulate the coded bits for source $S_{i}, i=1,2$, as follows:

$$
\hat{c}_{i, k}=\arg \min _{c_{i, k} \in\{0,1\}} \mathcal{M}\left(x_{i, k}\right), 1 \leq k \leq N,
$$

where the detection metric $\mathcal{M}\left(x_{i, k}\right)=\mid y_{S_{i} D, k}-$ $\left.\sqrt{P_{S_{i} D}} h_{S_{i} D x_{i, k}}\right|^{2}$ if $k \notin \Theta$; otherwise

$$
\begin{aligned}
\mathcal{M}\left(x_{i, k}\right) & =\left|y_{S_{i} D, k}-\sqrt{P_{S_{i} D}} h_{S_{i} D} x_{i, k}\right|^{2}+\lambda_{R_{i}^{\star}} \mid y_{R_{i}^{\star} D, k} \\
& -\left.\sqrt{P_{R_{i}^{\star} D}} h_{R_{i}^{\star} D^{\star}} \hat{x}_{R_{i}^{\star}, k}\right|^{2} .
\end{aligned}
$$

In (7), $\lambda_{R_{i}^{\star}}$ is the parameter of the C-MRC detector which is computed as $\lambda_{R_{i}^{\star}} \triangleq \frac{\min \left(\gamma_{S_{i} R_{i}^{\star}, \gamma_{R_{i}^{\star} D}}\right)}{\gamma_{R_{i}^{\star} D}}$, where $\gamma_{X Y}=$ $P_{X Y}\left|h_{X Y}\right|^{2} / \sigma^{2}$ being the instantaneous SNR of the channel $X \rightarrow Y$. The C-MRC detector then computes loglikelihood ratio values of the coded bits and sends them to the channel decoder. Finally, the channel decoder applies the BCJR algorithm [26] to decode the transmitted data.

Remark 1 In our protocol, the selected relay always forwards the estimated symbols to the destination. Fortunately, possible decoding error in $\hat{c}_{R_{i}^{\star}}$, , hence error propagation, is effectively mitigated by $\lambda_{R_{i}^{\star}}$ in C-MRC. For example, if the source-relay channel is too noisy, i.e., $\gamma_{S_{i} R_{i}^{\star}}$ is too small, it is highly probable that $\mathrm{R}_{i}^{\star}$ decodes with errors. In this case, however, $\lambda_{R_{i}^{\star}}$ is small and the contribution of the relayed signal is negligible.

\section{Relay selection for NCC and PARC}

In this section, we describe in details the relay selection process in NCC and PARC. Furthermore, essential properties of the selected relay channels are analyzed.

\subsection{Relay selection in NCC}

The RS process in NCC is performed based on a criterion that minimizes possible error of network-coded symbols. Because an error of the network-coded signal can result from either source-relay links or relay-destination link, the network-coded symbols can be seen as if it has been transmitted via an equivalent channel which yields the same error probability [20]. We remind that $\gamma_{X Y}$ denotes the instantaneous channel gain of the link $\mathrm{X} \rightarrow \mathrm{Y}$. By using the equivalent error probability for network-coded symbols, the two-hop source-relay-destination channel corresponding to the relay $\mathrm{R}_{j}$ can be tightly modeled as follows [20]:

$$
\gamma_{e q, j}=\min \left\{\gamma_{S_{1} R_{j}}, \gamma_{S_{2} R_{j}}, \gamma_{R_{j} D}\right\} .
$$

Because $\gamma_{S_{1} R_{j}}, \gamma_{S_{2} R_{j}}$ and $\gamma_{R_{j} D}$ are exponential random variables, it is straightforward to show that $\gamma_{e q, j}$ is also an 
exponential random variable. Denote $\bar{\gamma}_{(.)}=\mathbb{E}\left\{\gamma_{(.)}\right\}$as the mean of $\gamma_{(.)}$, where $\mathbb{E}\{\}$ is the expectation over the fading channels. The mean of the equivalent SNR $\gamma_{e q, j}$ is given as

$$
\frac{1}{\bar{\gamma}_{e q, j}}=\frac{1}{\bar{\gamma}_{S_{1} R_{j}}}+\frac{1}{\bar{\gamma}_{S_{2} R_{j}}}+\frac{1}{\bar{\gamma}_{R_{j} D}} \text {. }
$$

The best relay in NCC, $\mathrm{R}_{N C}$, is selected by the max-min criterion as follows:

$$
\mathrm{R}_{N C}=\arg \max _{R_{j}} \gamma_{e q, j}, 1 \leq j \leq N_{r} .
$$

The equivalent network-coded channel of the selected relay is given as

$$
\gamma_{N C}=\max \left\{\gamma_{e q, 1}, \ldots, \gamma_{e q, N_{r}}\right\} .
$$

Because the $\gamma_{e q, j}$ are mutually independent, the cumulative density function (CDF) of $\gamma_{N C}$ is computed as: $F_{\gamma_{N C}}(\gamma)=\prod_{j=1}^{N_{r}} F_{\gamma_{e q, j}}(\gamma)$. Taking the derivative of $F_{\gamma_{N C}}(\gamma)$ (with respect to $\gamma$ ), we obtain the probability density function (PDF) of $\gamma_{N C}$ expressed in the simplified form as follows:

$f_{\gamma_{N C}}(\gamma)=\sum_{j=1}^{N_{r}}\left((-1)^{j-1} \sum_{\substack{n_{1}=1, \ldots, n_{j}=1 \\ n_{1} \neq \cdots \neq n_{j}}}^{N_{r}} \frac{1}{\bar{\gamma}_{N C, j}} \exp \left(-\frac{\gamma}{\bar{\gamma}_{N C, j}}\right)\right)$, where $n_{1} \neq \cdots \neq n_{j}$ means $n_{1}, \ldots, n_{j}$ are all different and

$$
\frac{1}{\bar{\gamma}_{N C, j}}=\sum_{k=n_{1}}^{n_{j}}\left(\frac{1}{\bar{\gamma}_{S_{1} R_{k}}}+\frac{1}{\bar{\gamma}_{S_{2} R_{k}}}+\frac{1}{\bar{\gamma}_{R_{k} D}}\right) \text {. }
$$

The moment generating function (MGF) of $\gamma_{N C}$ is calculated as follows:

$$
\Psi_{\gamma_{N C}}(s)=\sum_{j=1}^{N_{r}}\left((-1)^{j-1} \sum_{\substack{n_{1}=1, \ldots, n_{j}=1 \\ n_{1} \neq \cdots \neq n_{j}}}^{N_{r}} \frac{1}{1+\bar{\gamma}_{N C, j} s}\right) .
$$

\subsection{Relay selection in PARC}

The selection process is executed at the beginning of each block in a distributed manner based on the max-min criterion that maximizes the worst end-to-end SNR and reduces computational complexity [6]. After the channel estimation, the relays set a timer that is inversely proportional to their channel gain. The first relay whose timer is zero will send a pulse to the destination. Upon receiving the pulse, the destination declares the chosen relay [3]. It is observed that the end-to-end performance of relayed symbols is determined by the weaker between sourcerelay and relay-destination connections. We thus model a two-hop source-relay-destination link by an equivalent single-hop channel, which is highly accurate for DMF relaying protocol [3].
The two-hop $\mathrm{S}_{i} \rightarrow \mathrm{R}_{j} \rightarrow \mathrm{D}$ channel is well modeled by $\gamma_{i j}=\min \left\{\gamma_{S_{i} R_{j}}, \gamma_{R_{j} D}\right\}, 1 \leq j \leq N_{r}$. Since both $\gamma_{S_{i} R_{j}}$ and $\gamma_{R_{j} D}$ are exponential random variables with mean $\bar{\gamma}_{S_{i} R_{j}}$ and $\bar{\gamma}_{R_{j} D}$, respectively, it is straightforward to show that $\gamma_{i j}$ is also an exponential random variable with mean $\bar{\gamma}_{i j}=\frac{\bar{\gamma}_{S_{i} R_{j}} \bar{\gamma}_{R_{j} D}}{\bar{\gamma}_{S_{i} R_{j}}+\bar{\gamma}_{R_{j} D}}, i=1,2$. The best relay $\mathrm{R}_{i}^{\star}$ for source $\mathrm{S}_{i}$ is selected to achieve the largest equivalent channel as follows:

$$
\mathrm{R}_{i}^{\star}=\arg \max _{R_{j}} \gamma_{i j}, 1 \leq j \leq N_{r} .
$$

The equivalent channel of the selected relay $R_{i}^{\star}, i=1,2$, is given as

$$
\gamma_{R_{i}^{\star}}=\max \left\{\gamma_{i 1}, \ldots, \gamma_{i N_{r}}\right\} .
$$

By using the max function [27], the PDF of $\gamma_{R_{i}^{\star}}$ is given in a shortened form as follows:

$f_{\gamma_{i}^{*}}(\gamma)=\sum_{j=1}^{N_{r}}\left((-1)^{j-1} \sum_{\substack{n_{1}=1, \ldots, n_{j}=1 \\ n_{1} \neq \cdots \neq n_{j}}}^{N_{r}} \frac{1}{\bar{\gamma}_{R_{i}^{\star}, j}} \exp \left(-\frac{\gamma}{\bar{\gamma}_{R_{i}^{\star}, j}}\right)\right)$, where $\frac{1}{\overline{\gamma_{R_{i}^{\star}, j}}}=\sum_{k=n_{1}}^{n_{j}}\left(\frac{1}{\overline{\gamma_{S_{i} R_{k}}}}+\frac{1}{\overline{\gamma_{R_{k}} D}}\right)$.

The MGF of $\gamma_{R_{i}^{\star}}$ is given as

$$
\Psi_{\gamma_{R_{i}^{\star}}}(s)=\sum_{j=1}^{N_{r}}\left((-1)^{j-1} \sum_{\substack{n_{1}=1, \ldots, n_{j}=1 \\ n_{1} \neq \cdots \neq n_{j}}}^{N_{r}} \frac{1}{1+\bar{\gamma}_{R_{i}^{\star}, j} s}\right) .
$$

Remark 2 The relay selection process in PARC is performed for each source separately, which is different from NCC. Also, the decoding process at the destination is performed separately for each source.

\section{Performance analysis for network coding-based cooperation}

In this section, we analyze the BER and diversity order of NCC. Using the equivalent channel, the two-hop networkcoded signal can be modeled as if it was conveyed by a single channel whose instantaneous SNR is $\gamma_{\mathrm{NC}}[20]$.

\subsection{Bit error rate analysis}

Recalling that in NCC, the destination applies the BCJR algorithm on the compound code $\mathbf{G}$, which is described in Section 2.1. The compound code $\mathbf{G}$ has the compound input $\mathbf{X}=\left[\mathbf{x}_{1}, \mathbf{x}_{2}, \mathbf{x}_{N C}\right]$ and the channel output $\mathbf{Y}=\left[\mathbf{y}_{S_{1} D}, \mathbf{y}_{S_{2} D}, \mathbf{y}_{R_{N C} D}\right]$. Note that the output of $\mathbf{G}$ undergoes some block fading channels with three blocks $\gamma_{S_{1} D}, \gamma_{S_{2} D}$, and $\gamma_{N C}$, and that $\mathbf{G}$ decodes the source data 
simultaneously. Consider $\mathbf{G}$ as a regular convolutional code, the BER of source $S_{i}$ is calculated as follows:

$$
\mathrm{BER}_{i}^{\mathrm{NC}}=\frac{1}{2} \sum_{d=F}^{+\infty} w_{i}(d) \overline{\operatorname{PEP}}^{\mathrm{NC}}(d), i=1,2,
$$

where $F$ is the minimum distance of the compound code G, $w_{i}(d)$ denotes input weights (number of non-zero information bits) corresponding to source $S_{i}$ in the compound codeword, and $\overline{\mathrm{PEP}}^{\mathrm{NC}}(d)$ is the unconditioned pair-wise error probability (UPEP) $)^{3}$ of receiving a compound codeword with the output weight $d$ (number of non-zero coded bits), assuming that the all-zero codeword, e.g., $\mathbf{c}_{1}=\mathbf{c}_{2}=$ 0, has been transmitted. To derive (10), it requires the knowledge of the minimum distance $F$ of the compound code, the input weight $w_{i}(d)$, and how $d$ output wrights in the compound codeword $\mathbf{X}$ are distributed among the three channels $\mathrm{S}_{1} \rightarrow \mathrm{D}, \mathrm{S}_{2} \rightarrow \mathrm{D}$ and $\mathrm{R}_{N C} \rightarrow \mathrm{D}$. Denote $\mathbf{W}_{d}=\left\{d_{1}, d_{2}, d_{R}\right\}$ as the weight pattern that specifies how $d$ weights are distributed among these channels, where $d_{i}$ is the output weight of the individual codeword transmitted via the channel $\mathrm{S}_{i} \rightarrow \mathrm{D}$ or channel $\mathrm{R}_{N C} \rightarrow \mathrm{D}$. By definition, $d=d_{1}+d_{2}+d_{R}$. The input weight and the pattern can be computed via heuristic searching of the trellis of G. The following result is important for further analysis:

Lemma 1 The minimum distance $F$ of the compound code $\mathbf{G}$ is equal to twice the minimum distance $f$ of the single code $\mathbf{g}, F=2 f$, and the weight pattern $\mathbf{W}_{F}$ has one of the following values $\{f, f, 0\},\{f, 0, f\},\{0, f, f\}$.

Lemma 2 For any pattern $\mathbf{W}_{d}=\left\{d_{1}, d_{2}, d_{R}\right\}$ of the compound codeword $\mathbf{X}$ with output weight $d>F$, there are at least two non-zero elements in $\mathbf{W}_{d}$.

The proof of Lemma 1 and Lemma 2 are given in [21]. Lemma 1 and Lemma 2 provide an important information about the output weights of the compound code: $d$ weights of the compound code always experience at least two independent channels. Furthermore, the number of patterns is finite and strictly defined by $\mathbf{G}$.

By using Lemma 1 and 2, we can reformulate (10) as follows:

$$
\mathrm{BER}_{i}^{\mathrm{NC}}=\frac{1}{2} \sum_{d=F}^{+\infty} \sum_{\mathbf{W}_{d}} w_{i}\left(\mathbf{W}_{d}\right) \overline{\operatorname{PEP}}^{\mathrm{NC}}\left(d \mid \mathbf{W}_{d}\right),
$$

where $\overline{\mathrm{PEP}}^{\mathrm{NC}}\left(d \mid \mathbf{W}_{d}\right)$ is the UPEP depending on the pattern $\mathbf{W}_{d}$ and is the expectation of the conditioned pairwise error probability (CPEP) over the fading channels:

$$
\overline{\operatorname{PEP}}^{\mathrm{NC}}\left(d \mid \mathbf{W}_{d}\right)=\mathbb{E}\left\{\operatorname{PEP}^{\mathrm{NC}}\left(d \mid \mathbf{W}_{d}\right)\right\} .
$$

It is assumed that the erroneous detected symbol is one of the nearest neighbor symbols. Using the Gray mapping, each closest symbol error only causes one coded bit error. Therefore, the CPEP $\operatorname{PEP}^{\mathrm{NC}}\left(d \mid \mathbf{D}_{d}\right)$ is approximated as [21]:

$$
\operatorname{PEP}^{\mathrm{NC}}\left(d \mid \mathbf{W}_{d}\right)=Q\left(\sqrt{2 \gamma_{\Sigma_{N C}}}\right),
$$

where $\gamma_{\Sigma_{N C}}=d_{1} \gamma_{S_{1} D}+d_{2} \gamma_{S_{2} D}+d_{R} \gamma_{N C}$ is defined as the total SNR at the destination in NCC, and $Q(x)=$ $\frac{1}{\sqrt{2 \pi}} \int_{x}^{+\infty} e^{-t^{2} / 2} d t$ denotes the Q-function.

Because the three channels in $\gamma_{\Sigma_{N C}}$ are mutually independent, the MGF of $\gamma_{\Sigma_{N C}}$ can be computed as follows:

$$
\Psi_{\gamma_{\Sigma_{N C}}}(s)=\Psi_{\gamma_{S_{1} D}}\left(d_{1} s\right) \times \Psi_{\gamma_{S_{2} D}}\left(d_{2} s\right) \times \Psi_{\gamma_{N C}}\left(d_{R} s\right) .
$$

Applying the MGF method [27] we can derive the UPEP $\overline{\operatorname{PEP}}^{\mathrm{NC}}\left(d \mid \mathbf{W}_{d}\right)$ in NCC as in Theorem 1.

Theorem 1 Given the weight pattern $\mathbf{W}_{d}=\left\{d_{1}, d_{2}, d_{R}\right\}$, $d=d_{1}+d_{2}+d_{R}$, the UPEP $\overline{P E P}^{N C}\left(d \mid \mathbf{W}_{d}\right)$ of the compound code in NCC has a form given by:

$$
\overline{\operatorname{PEP}}^{N C}\left(d \mid \mathbf{W}_{d}\right)= \begin{cases}\mathcal{I}_{1}\left(d_{1} \bar{\gamma}_{S_{1} D}, d_{2} \bar{\gamma}_{S_{2} D}\right), & \text { if } d_{R}=0 \\ \sum_{j=1}^{N_{r}}(-1)^{j-1} \Omega_{1}, & \text { if } d_{1}=0 \\ \sum_{j=1}^{N_{r}}(-1)^{j-1} \Omega_{2}, & \text { if } d_{2}=0 \\ \sum_{j=1}^{N_{r}}(-1)^{j-1} \Omega_{3}, & \text { if } d_{1} d_{2} d_{R} \neq 0\end{cases}
$$

where

$$
\begin{aligned}
& \Omega_{1}=\sum_{\substack{n_{1}=1, \ldots, n_{j}=1 \\
n_{1} \neq \cdots \neq n_{j}}}^{N_{r}} \mathcal{I}_{1}\left(d_{2} \bar{\gamma}_{S_{2} D}, d_{R} \bar{\gamma}_{N C, j}\right), \\
& \Omega_{2}=\sum_{\substack{n_{1}=1, \ldots, n_{j}=1 \\
n_{1} \neq \cdots \neq n_{j}}}^{N_{r}} \mathcal{I}_{1}\left(d_{1} \bar{\gamma}_{S_{1} D}, d_{R} \bar{\gamma}_{N C, j}\right), \\
& \Omega_{3}=\sum_{\substack{n_{1}=1, \ldots, n_{j}=1 \\
n_{1} \neq \cdots \neq n_{j}}}^{N_{r}} \mathcal{I}_{2}\left(d_{1} \bar{\gamma}_{S_{1} D}, d_{2} \bar{\gamma}_{S_{2} D}, d_{R} \bar{\gamma}_{N C, j}\right),
\end{aligned}
$$

and

$$
\begin{aligned}
\mathcal{I}_{1}(a, b)= & \frac{1}{2}\left(1-\frac{a}{a-b} \sqrt{\frac{a}{a+1}}-\frac{b}{b-a} \sqrt{\frac{b}{b+1}}\right), \\
\mathcal{I}_{2}(a, b, c)= & \frac{1}{2}\left(1-\frac{a^{2}}{(a-b)(a-c)} \sqrt{\frac{a}{a+1}}\right. \\
& \left.-\frac{b^{2}}{(b-a)(b-c)} \sqrt{\frac{b}{b+1}}-\frac{c^{2}}{(c-a)(c-b)} \sqrt{\frac{c}{c+1}}\right) .
\end{aligned}
$$




\section{Proof See Appendix A.}

It is worth noting in (11) that the BER of each source in NCC is a sum of terms given in Theorem 1, weighted by their corresponding input weights $\mathrm{w}_{i}\left(\mathbf{W}_{d}\right)$. In $\mathrm{NCC}$, the weight pattern only holds a few values, and the input weights are computed from the extended distance spectrum. Table 1 gives an example of the distance spectrum of the compound code.

\subsection{Diversity analysis}

Since the BER is linearly proportional to the UPEP $\overline{\mathrm{PEP}}^{\mathrm{NC}}\left(d \mid \mathbf{W}_{d}\right)$ via corresponding input weights, the diversity order of NCC is equal to diversity order of the UPEP. Let $x \propto \bar{\gamma}^{-\eta}$ denote the exponential equivalence, i.e., $x$ achieves diversity of order $\eta$, where $\bar{\gamma}$ stands for the general average SNR. The diversity order of the $\overline{\mathrm{PEP}}^{\mathrm{NC}}\left(d \mid \mathbf{W}_{d}\right)$ is given by the following theorem.

Theorem 2 Given the weight pattern $\mathbf{W}_{d}=\left\{d_{1}, d_{2}, d_{R}\right\}$ with $d=d_{1}+d_{2}+d_{R}$, the UPEP $\overline{P E P}^{N C}\left(d \mid \mathbf{W}_{d}\right)$ in NCC has an exponential equivalent form as follows:

$$
\overline{\operatorname{PEP}}^{N C}\left(d \mid \mathbf{W}_{d}\right) \propto \begin{cases}\bar{\gamma}^{-2}, & \text { if } d_{R}=0 \\ \bar{\gamma}^{-\left(N_{r}+1\right)}, & \text { if } d_{1}=0 \text { or } d_{2}=0 . \\ \bar{\gamma}^{-\left(N_{r}+2\right)}, & \text { if } d_{1} d_{2} d_{R} \neq 0\end{cases}
$$

Proof See Appendix B.

It is shown from (11) and Theorem 2 that the BER in NCC is a combination of three factors whose respective diversity orders are $2, N_{r}+1$ and $N_{r}+2$. As the contribution of these factors are comparable and equal to the input weights of the compound code (shown in Table 1 as an example), the diversity order of NCC is dominated by the diversity order 2 factor. Consequently, NCC achieves diversity order 2 regardless of the channel code and the total number of available relays. This result is in line with the diversity order of NCC obtained via outage analysis $[11,12]$.

\section{Performance analysis for partial relaying-based cooperation}

In this section, we analyze the BER and diversity order of PARC by using the equivalent channel model. Since

Table 1 Input weight and output weight distribution at $d=F=24$ of compound code $\mathbf{G}$ in (4), $\mathbf{g}=[23,35,37]$

\begin{tabular}{lllll}
\hline$w_{1}$ & $w_{2}$ & $d_{1}$ & $d_{2}$ & $d_{R}$ \\
\hline 0 & 12 & 0 & 12 & 12 \\
12 & 0 & 12 & 0 & 12 \\
12 & 12 & 12 & 12 & 0 \\
\hline
\end{tabular}

PARC is symmetric, the analysis for two sources is similar. For ease of presentation, we drop the source subscript in this section. After two phases, the destination receives two signals from the source $S$ and the selected relay $R^{\star}$. The combined signal at the C-MRC detector's output can be classified into two groups: the first group consists of symbols which are helped by the selected relay, and the second group includes the rest of the symbols which are not relayed. In other words, the received signal at the destination can be seen as an output of a block fading channel with two blocks: one block consisting of the $N / 2$ symbols which only see channel $\gamma_{S D}$, and the other one containing the other $N / 2$ symbols which see both channel $\gamma_{S D}$ and $\gamma_{R^{\star}}$.

\subsection{Bit error rate analysis}

The destination applies the BCJR algorithm on the CC $\mathbf{g}$ to decode for the source data. Let $\overline{\mathrm{PEP}}(d)$ be the UPEP, which is the probability that the destination correctly decodes a codeword with Hamming weight $d$ (number of non-zero coded bits in $\mathbf{c}$ at the source) when the all-zero codeword was transmitted. The BER of PARC is upper-bounded as follows [28]:

$$
\mathrm{BER}^{\mathrm{PA}} \leq \sum_{d=f}^{N} w(d) \overline{\mathrm{PEP}}^{\mathrm{PA}}(d)
$$

where $f$ is the minimum distance of the channel code, and $w(d)$ are the input weights which are computed directly from the structure of the $\mathrm{CC}$ g. The $\overline{\mathrm{PEP}}^{\mathrm{PA}}(d)$ is the expectation over the channel fading coefficients of the CPEP PEP $\mathrm{PA}^{\mathrm{PA}}(d)$ as $\overline{\mathrm{PEP}}^{\mathrm{PA}}(d)=\mathbb{E}\left\{\mathrm{PEP}^{\mathrm{PA}}(d)\right\}$. Obviously, $\operatorname{PEP}^{\mathrm{PA}}(d)$ depends on the channel fading coefficients and the weight pattern $\mathbf{D}_{d}=\left\{d_{1}, d_{2}\right\}, d_{1}+d_{2}=d$, which specifies how the $d$ non-zero coded bits are distributed within the two blocks $\left(\gamma_{S D}\right.$ and $\left.\gamma_{S D}+\gamma_{R^{\star}}\right)$. Because the $d$ non-zero coded bits are uniformly distributed in the two blocks, the CPEP can be further analyzed as follows:

$$
\operatorname{PEP}^{\mathrm{PA}}(d)=\sum_{\mathbf{D}_{d}} \operatorname{PEP}^{\mathrm{PA}}\left(d \mid \mathbf{D}_{d}\right) p\left(\mathbf{D}_{d}\right)
$$

where $p\left(\mathbf{D}_{d}\right)$ is the probability of pattern $\mathbf{D}_{d}$, which is computed by combinatoric computation as

$$
p\left(\mathbf{D}_{d}\right)=\frac{\mathcal{C}_{d_{1}}^{N / 2} \times \mathcal{C}_{d_{2}}^{N / 2}}{\mathcal{C}_{d}^{N}}
$$

where $\mathcal{C}_{k}^{n}=\frac{n !}{(n-k) ! \times k !}$.

Substituting (14) into $\overline{\mathrm{PEP}}^{\mathrm{PA}}(d)$, we obtain

$$
\overline{\operatorname{PEP}}^{\mathrm{PA}}(d)=\sum_{\mathbf{D}_{d}} \underbrace{\mathbb{E}\left\{\operatorname{PEP}^{\mathrm{PA}}\left(d \mid \mathbf{D}_{d}\right)\right\}}_{\overline{\mathrm{PEP}}^{\mathrm{PA}}\left(d \mid \mathbf{D}_{d}\right)} p\left(\mathbf{D}_{d}\right) .
$$


Given the pattern $\mathbf{D}_{d}=\left\{d_{1}, d_{2}\right\}$, there are $d_{1}$ nonzero coded bits carried by block $\gamma_{S D}$ and $d_{2}$ non-zero coded bits carried by block $\gamma_{S D}+\gamma_{R^{\star}}$. As a result, the $\mathrm{CPEP} \mathrm{PEP}_{\mathrm{PA}}\left(d \mid \mathbf{D}_{d}\right)$ is calculated using similar techniques in [29], as follows:

$$
\operatorname{PEP}^{\mathrm{PA}}\left(d \mid \mathbf{D}_{d}\right)=Q\left(\sqrt{2 \gamma_{\Sigma}}\right)
$$

where $\gamma_{\Sigma}=d_{1} \gamma_{S D}+d_{2}\left(\gamma_{S D}+\gamma_{R^{\star}}\right)=d \gamma_{S D}+d_{2} \gamma_{R^{\star}}$.

Taking into account the independence between $\gamma_{S D}$ and $\gamma_{R^{\star}}$, we have $\Psi_{\gamma_{\Sigma}}(s)=\Psi_{\gamma_{S D}}(d s) \times \Psi_{\gamma_{R^{\star}}}\left(d_{2} s\right)$.

Theorem 3 Given the weight pattern $\mathbf{D}_{d}=\left\{d_{1}, d_{2}\right\}, d=$ $d_{1}+d_{2}$, the UPEP $\overline{P E P}^{P A}\left(d \mid \mathbf{D}_{d}\right)$ of PARC is calculated as follows:

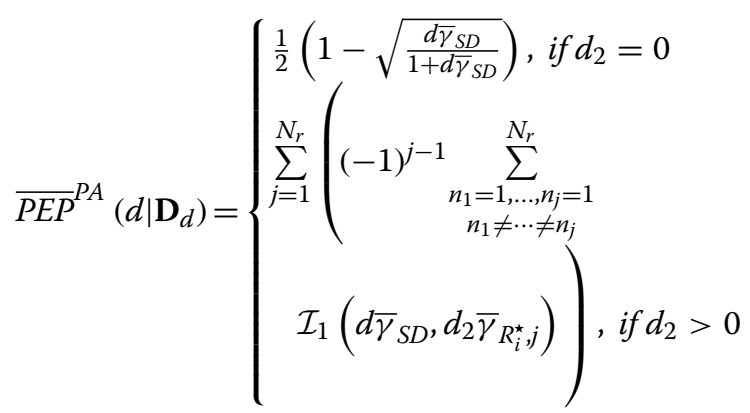

where $\mathcal{I}_{1}(a, b)$ has been defined in Theorem 1.

Proof See Appendix C.

Substituting $\overline{\mathrm{PEP}}^{\mathrm{PA}}\left(d \mid \mathbf{D}_{d}\right)$ from Theorem 3 into (16) and (13), we obtain the upper bound for the BER. Note that even though $d$ in (13) can be as large as the codeword's length, i.e., $N$, the BER usually depends on a few first values in fading channels. To give insightful understanding of PARC, we analyze the system diversity order.

\subsection{Diversity analysis for $\overline{\mathrm{PEP}}^{\mathrm{PA}}\left(d \mid \mathrm{D}_{d}\right)$}

We first analyze the diversity order of the UPEP $\overline{\mathrm{PEP}}^{\mathrm{PA}}\left(d \mid \mathbf{D}_{d}\right)$ for a given weight pattern $\mathbf{D}_{d}$, which determines how the selected relay contributes to the overall system performance.

Theorem 4 Given the weight pattern $\mathbf{D}_{d}=\left\{d_{1}, d_{2}\right\}$ with $d=d_{1}+d_{2}$, the UPEP $\overline{P E P}^{P A}\left(d \mid \mathbf{D}_{d}\right)$ in PARC has an exponential equivalent given as follows:

$$
\overline{P E P}^{P A}\left(d \mid \mathbf{D}_{d}\right) \propto\left\{\begin{array}{ll}
\bar{\gamma}^{-1}, & \text { if } d_{2}=0 \\
\bar{\gamma}^{-\left(N_{r}+1\right)}, & \text { if } d_{2}>0
\end{array},\right.
$$

where $\propto$ denotes the proportional relation.
Proof See Appendix D.

Theorem 4 states that $\overline{\mathrm{PEP}}^{\mathrm{PA}}\left(d \mid \mathbf{D}_{d}\right)$ can have either diversity order one or diversity order of $N_{r}+1$ depending on the weight pattern $\mathbf{D}_{d}$.

\subsection{Diversity analysis of PARC}

This subsection analyzes the diversity order of the proposed PARC scheme. It is observed from (13) that the diversity order of PARC is determined by $\overline{\mathrm{PEP}}^{\mathrm{PA}}(d)$ because the input weight $w(d)$ of the channel code is constant. From (16), we conclude that $\overline{\mathrm{PEP}}^{\mathrm{PA}}(d)$ is a combination of one factor with diversity of order one (corresponding to the pattern $D_{1} \triangleq(d, 0)$ ) and one factor with diversity of order $N_{r}+1$. The contribution of the factor with diversity order one is computed from (15) as

$$
p\left(D_{1}\right)=\frac{\mathcal{C}_{d}^{N / 2}}{\mathcal{C}_{d}^{N}}=\prod_{k=0}^{d-1} \frac{N-2 k}{2 N-2 k} .
$$

In practices, the codeword length $N$ is usually much larger than $d$, then $p\left(D_{1}\right)$ can be well-approximated as

$$
p\left(D_{1}\right) \simeq\left(\frac{1}{2}\right)^{d} \leq\left(\frac{1}{2}\right)^{f} .
$$

From (18), (13), and Theorem 4, we have

$$
\operatorname{BER}^{\mathrm{PA}}(\bar{\gamma})=K 2^{-f} \bar{\gamma}^{-1}+\bar{\gamma}^{-N_{r}-1},
$$

where $K$ is the normalized constant that depends on the channel code and network topology.

The classical definition of diversity order is defined as the negative exponent of the average BER as a function of SNR in log-log scale [32], which visually represents the slope of the BER curve at the high SNR regime. In this paper, since we are interested in the system behavior in the finite-SNR regime, we define the diversity order at an arbitrary (average) SNR $\bar{\gamma}$ as the slope of BER curve in the $\log$-log scale [30]:

$$
\begin{aligned}
\zeta(\bar{\gamma}) & \triangleq-\lim _{\Delta \rightarrow 0} \frac{\log (\operatorname{BER}(\bar{\gamma}+\Delta))-\log (\operatorname{BER}(\bar{\gamma}))}{\log (\bar{\gamma}+\Delta)-\log (\bar{\gamma})} \\
& =-\left.\gamma \frac{\partial \log (\operatorname{BER}(\gamma))}{\partial \gamma}\right|_{\gamma=\bar{\gamma}},
\end{aligned}
$$

which obviously matches the classical definition of diversity when the SNR tends to infinity. Because the diversity order depends on the SNR, we refer to $\zeta(\bar{\gamma})$ as instantaneous diversity order. The key idea behind the definition is that it allows the system behavior to be studied at any SNR value.

Substituting (19) into (20), we obtain the instantaneous diversity order of PARC as follows:

$$
\zeta(\bar{\gamma})=1+\frac{N_{r}}{1+K 2^{-f} \bar{\gamma}^{N_{r}}} .
$$


An important observation from (21) is that the instantaneous diversity order of PARC depends on the operating SNR value and the channel code, which provides a criterion design to achieve desirable diversity order in the finite-SNR regime. By choosing a proper channel code whose minimum distance $f$, such as $K 2^{-f} \bar{\gamma}_{*}^{N_{r}} \ll 1$, then the PARC achieves full (instantaneous) diversity order of $N_{r}+1$ in the SNR region $\left[0, \bar{\gamma}_{*}\right]$. This result is crucial because the operating SNR regime is usually finite in practice.

\section{Numerical results}

This section presents simulation results to confirm the effectiveness of the proposed PARC. All channels are subject to quasi-static block Rayleigh fading plus AWGN. Because we focus on the diversity order, which is not affected by modulation order, BPSK modulation and binary network coding are carried out in simulations. The data packet length is equal to 1024 bits. We consider symmetric network, i.e., $\bar{\gamma}_{S_{i} R_{j}}=\bar{\gamma}_{S R}, \bar{\gamma}_{R_{j} D}=\bar{\gamma}_{R D}, \bar{\gamma}_{S_{i} D}=$ $\bar{\gamma}_{S D}, \forall i, j$. Unless otherwise stated, the relays are located in the middle of the sources and the destination. The path loss exponent is equal to 3.5. As a result, the average SNR in source-relay channels and relay-destination channels are $10.5 \mathrm{~dB}$ better than source-destination channels. Note that our analysis holds for arbitrary locations of the relays. The channel code is chosen as the one that optimizes both the minimum distance and distance spectrum in block Rayleigh fading channels [31]. Different channel codes $\mathbf{g}$ are compared.
We also present the performance of two reference schemes. The first reference scheme (named Reference 1 in the figures) is based on fractional repetition coding cooperation $[22,23]$. The second reference scheme employs fractional repetition coding together with network coding (named Reference 2 in the figures). All relays are active and share the relaying phase in two reference schemes. In Reference 1, since the relays help the sources separately, each relay forwards $1 /\left(2 N_{r}\right)$ of the estimated codeword. In Reference 2, all relays use NC to help the sources, and each relay randomly forwards $1 / N_{r}$ of the network-coded codeword. Note that no relay selection is used in the reference schemes.

Figure 3 compares the bounds derived in Theorem 1 and Theorem 3 with the simulations. The first six output weights are used to compute the bounds. It is shown that the derived bounds are consistent with simulation results for both PARC and NCC, and for different channel codes, which demonstrates the accuracy of the analysis.

Figure 4 compares the performance of PARC and NCC with the references for the channel code [133 165 171] with code rate $1 / 3$ and minimum distance $f=15$. The total number of relays $N_{r}$ equal to 2 and 4 are plotted. The observed performance region satisfies BER $\geq 10^{-6}$ because this is the target BER for most practical applications. It is shown in the figure that the proposed PARC achieves an instantaneous diversity order of 3 and 5 (full diversity order) in the observed SNR range for $N_{r}=2$ and $N_{r}=4$, respectively. Such results can be explained from Theorem 2 that the impact of the diversity one factor

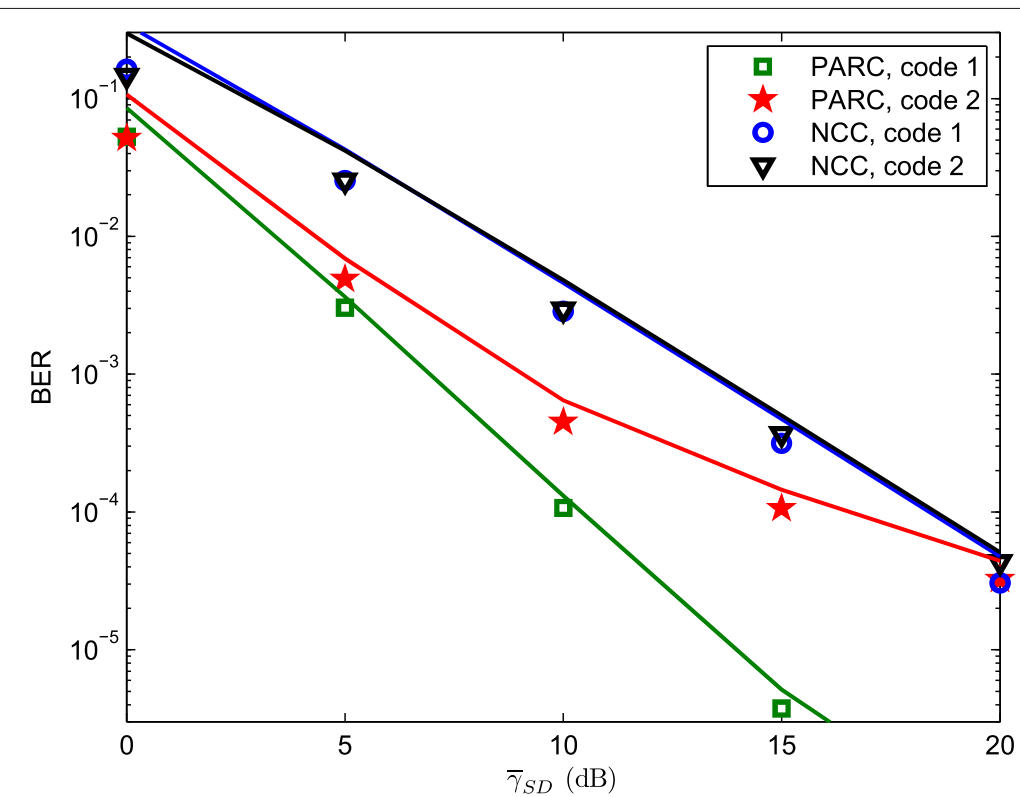

Fig. 3 Validation of the analysis for both PARC and NCC. Markers show simulation results, solid curves correspond to the bounds in (10) and (13). $N_{r}=2$, code 1: [133 165 171], code 2: [5 7 5] 


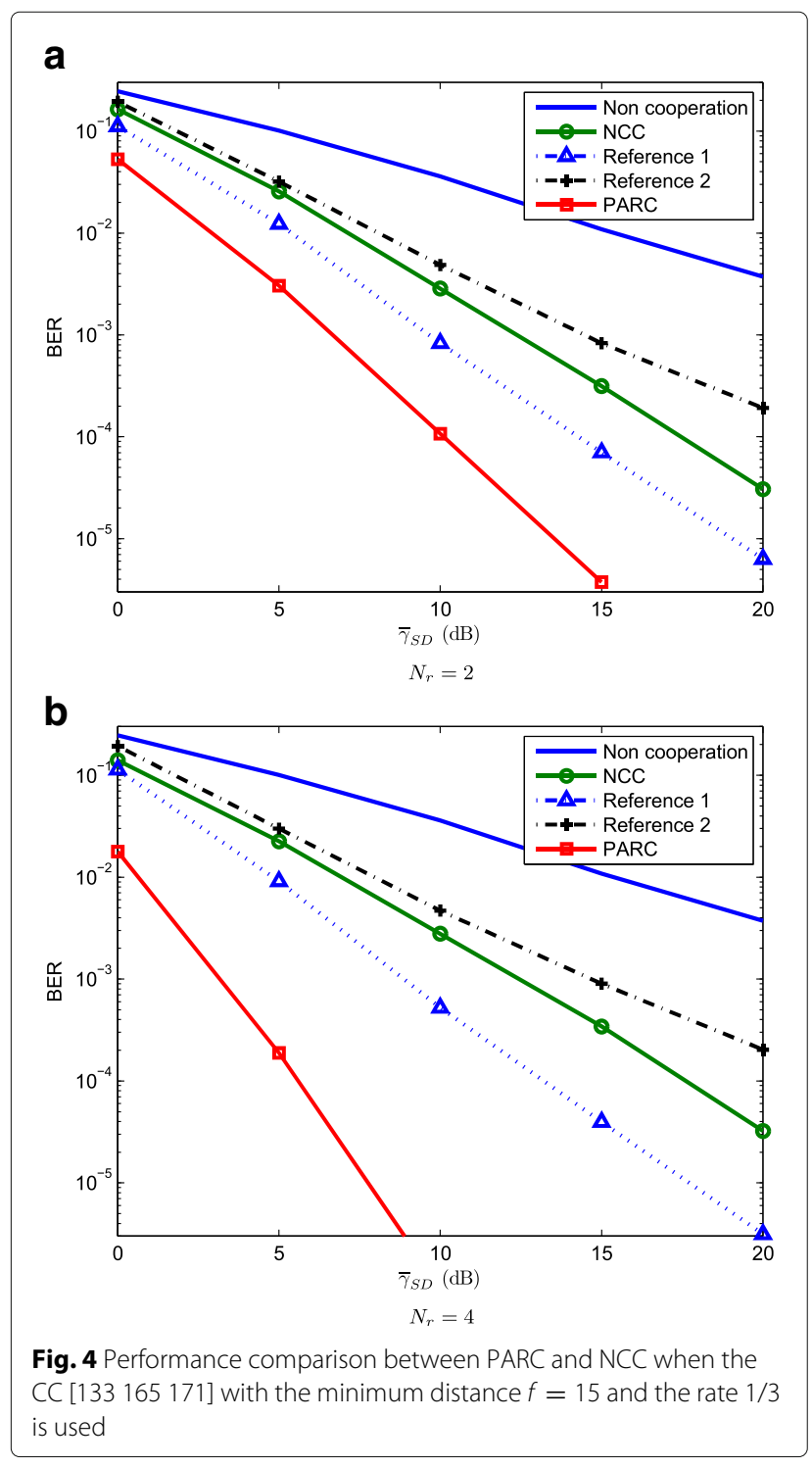

equals $p\left(\mathrm{D}_{1}\right)=(1 / 2)^{f} \simeq 3.10^{-5}$, which is negligible. Therefore, the diversity order of PARC is determined by the full diversity factor in the observed SNR region. In contrast, NCC always achieve a diversity of order 2, which is direct infer from Theorem 4. A huge SNR gain is therefore achieved by PARC. In particular, PARC outperforms all other schemes about $5 \mathrm{~dB}$ for $N_{r}=2$ and $10 \mathrm{~dB}$ for $N_{r}=4$ at $\mathrm{BER}=10^{-4}$. Another observation is that Reference 1 also surpasses NCC because the relayed symbols in Reference 1 see more spatial diversity gain than in NCC. When SNR tends to infinity, NCC may outperform PARC because the diversity order of PARC will eventually collapse to one while NCC still has diversity of order 2. From the practical system point of view, this crossingpoint might not weaken the advantage of PARC over NCC since practical systems usually operate in the finite-SNR regime, and the inflection point in the BER curve may be located in very high SNR values, way above the nominal operating point of the system.

Figure 5 shows performance comparisons when the channel code [25 33 37] with rate $1 / 3$ is used. The minimum distance of this code is equal to 12 . Again, NCC still achieves diversity order 2 for both $N_{r}=2$ and $N_{r}=4$, and the performance of NCC in both cases is similar. It is observed that PARC only achieves full instantaneous diversity order in low SNRs. More specifically, PARC achieves diversity of order 3 in the SNR range until $10 \mathrm{~dB}$ for $N_{r}=2$ and diversity of order 5 until SNR $=5 \mathrm{~dB}$ for $N_{r}=4$. As SNR increases, a degradation in the instantaneous diversity order is observed, which is predicted by our analysis (for this code, the contribution of diversity order one factor approximately is $2^{-f} \simeq 2.4 e-4$ ). However, a similar SNR gain as in the strong code [133 165 171]

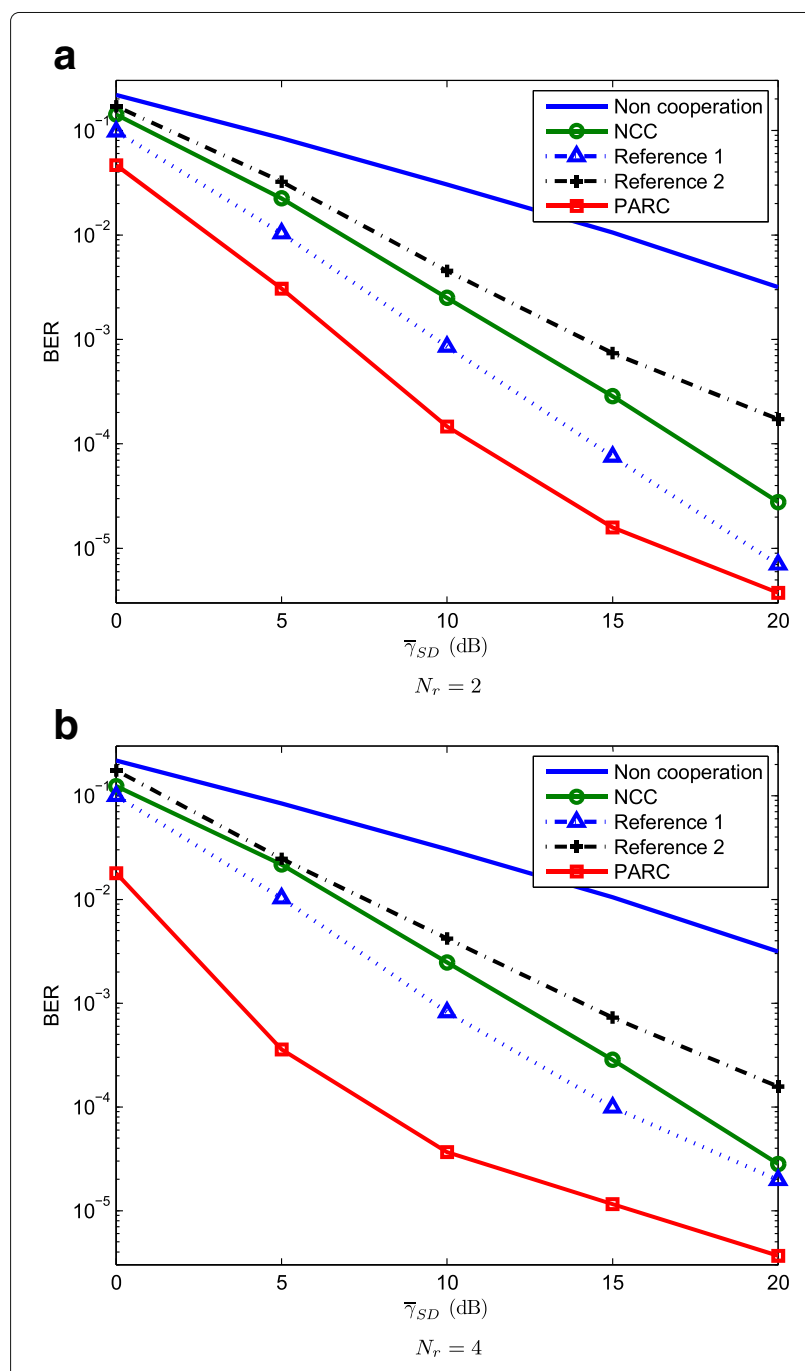

Fig. 5 Performance comparison between PARC and NCC when the CC [25 33 37] with the minimum distance $f=12$ and the rate $1 / 3$ is used 


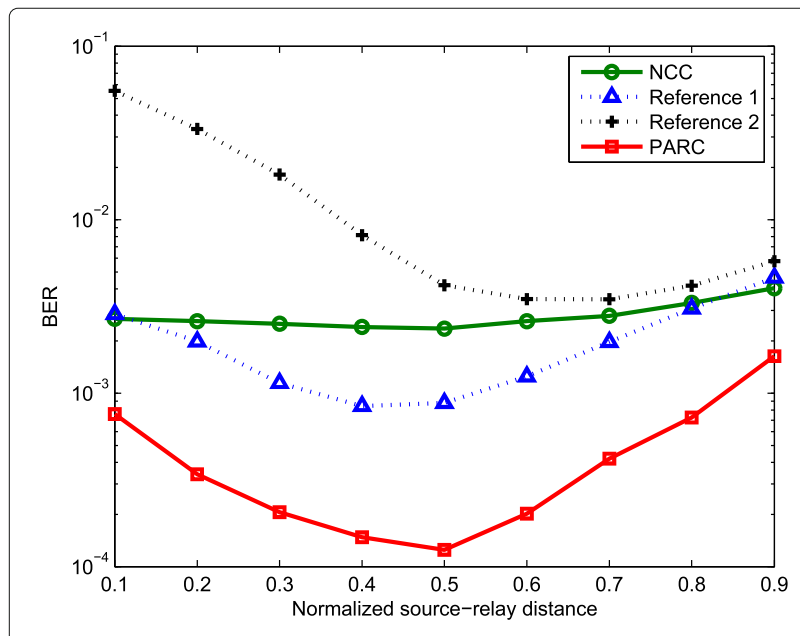

Fig. 6 Performance comparison between PARC and the references for various relay's locations. Normalized source-relay distance is measured by the source-relay distance divided by the source-destination distance. Channel code [25 33 37], SNR = $10 \mathrm{~dB}$

is achieved by PARC at BER of $10^{-4}$, which is about $5 \mathrm{~dB}$ for $N_{r}=2$ and $7 \mathrm{~dB}$ for $N_{r}=4$. An interesting observation is that the performance Reference 1 for $N_{r}=4$ is worse than that for $N_{r}=2$. This is because in the latter, the relay forwards fewer symbols for the case $N_{r}=4$ than the case $N_{r}=2$, and the channel code is not strong enough to compensate for this loss [29].

Figure 6 compares the BER of the proposed PARC with the references for various relay's locations, i.e., close to the sources toward the destination. It is shown that the proposed PARC significantly outperforms the references, and the relative relay's position has effects on all schemes. In particular, all schemes achieve the smallest BER when the relay is located around the middle between the sources and the destination. Moving closer to either the sources or destination results in higher BER. This is because in this case, either source-relay or relay-destination link is in poor condition, resulting in bad relayed signal since the cooperative MRC receiver weights the relayed signal based on the worse source-relay and relay-destination link.

Figure 7 compares the performance between PARC and NCC for various channel codes and $N_{r}=3$. Three codes with different error correction capabilities are presented: the weak code [ 57 5] with small minimum distance $f=7$, the moderate code [25 33 37] with $f=12$, and the strong code [133 165171 ] with $f=15$. Full diversity order is observed in low SNRs for all codes. When SNR increases, only the strong code achieve full diversity order in the observed SNR range. The weak code starts losing diversity order earliest at SNR of $5 \mathrm{~dB}$, while the moderate code's diversity order degrades at $10 \mathrm{~dB}$. Compared with NCC, however, PARC significantly outperforms the references for all codes in the observed SNRs.

In conclusion, the most effective of the proposed PARC is the capability of achieving full (instantaneous) diversity order in the low and medium SNR regime, which in turn results in a large SNR gain in the finite-SNR regime. Such gain is crucial for practical systems because their operating SNRs is usually finite.

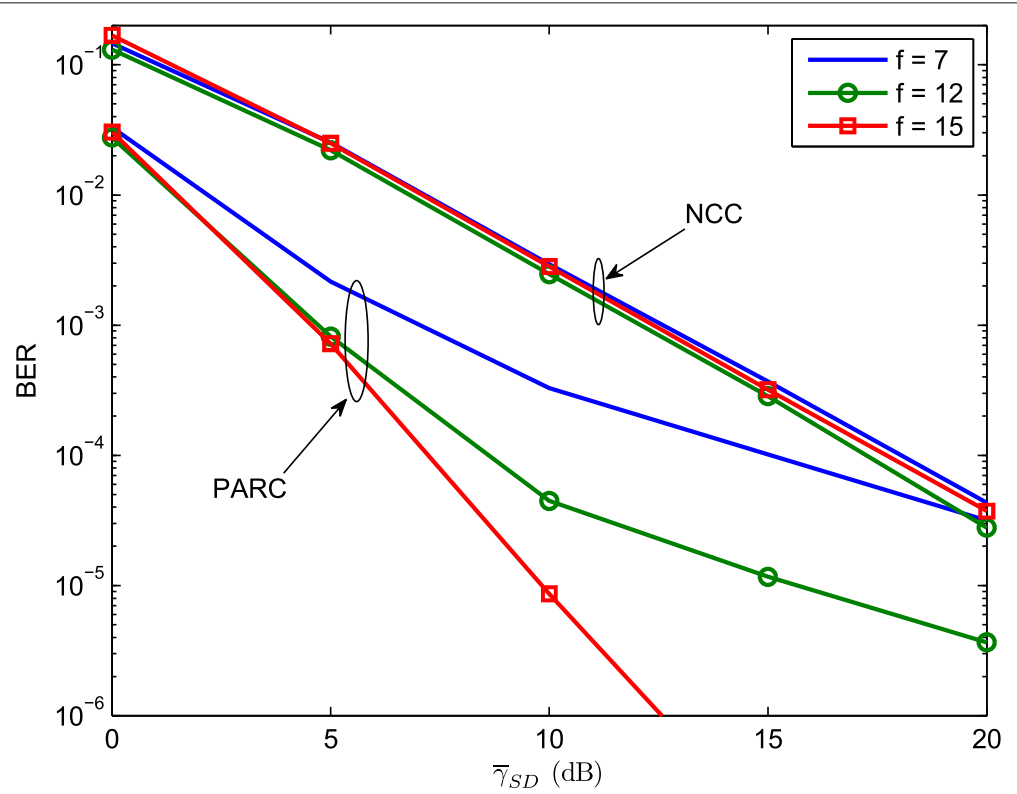

Fig. 7 Performance comparison between PARC and NCC for different minimum distances, which corresponds to code's correction capacity. Three codes with rate $1 / 3$ are compared: CC [5 7 5] with $f=7$, CC [25 33 37] with $f=12$, and CC [133 165 171] with $f=15$ 


\section{Conclusions}

We have proposed a novel cooperative scheme for a twosource multiple-relay network that combines the best relay selection and partial relaying cooperation to effectively exploit the spatial diversity. We have shown that the instantaneous diversity order is a function of the minimum distance of the channel code and the operating SNR. It has been shown by both analytical and simulation results that our proposed scheme can gain full diversity order in the finite-SNR regime when a suitable channel code is used.

The proposed scheme can easily be extended to general multi-source multi-relay networks. In this case, the selected relays might forward a number of symbols which is less than half of the codeword length. The major challenge in this case is how to select best (multiple) relays for network coding and partial relaying. A promising application of PARC is to design a cooperation scheme to support multiple sources with different error correction capacities to achieve a given target BER. This problem can be solved by carefully designing how many symbols of each source should be relayed depending on the corresponding channel code's strength.

\section{Endnotes}

${ }^{1}$ Instantaneous diversity order is measured as the slope of the BER curve in log-log scale, which allows to study the system behavior at arbitrary SNR value. This definition coincides with the conventional diversity definition in the high SNR regime [32]

${ }^{2}$ Other selection of $\Theta$, e.g., optimal index set, can be employed, but are beyond the scope of this paper.

3 The UPEP does not depend on the fading channels.

\section{Appendix A: proof of Theorem 1}

Recall that the total SNR in NCC is given by $\gamma_{\Sigma_{N C}}=$ $d_{1} \gamma_{S_{1} D}+d_{2} \gamma_{S_{2} D}+d_{R} \gamma_{N C}$. To derive the UPEP for NCC, the MGF method is employed. Lemma 1 and Lemma 2 state that there are at least two non-zero weights in $\left\{d_{1}, d_{2}, d_{R}\right\}$. Therefore, the weight pattern $\mathbf{W}_{d}$ can only be one of these four cases: 1) $\left.\left.d_{R}=0,2\right) d_{1}=0,3\right) d_{2}=0$, and 4 ) $d_{1} d_{2} d_{R} \neq 0$.

- Case 1: $d_{R}=0$, there is not any weight on the channel $\gamma_{N C}$. The total SNR is equal to $\gamma_{\Sigma_{N C}}=d_{1} \gamma_{S_{1} D}+d_{2} \gamma_{S_{2} D}$, and its MGF is given as follows:

$$
\begin{aligned}
\Psi_{\gamma_{\Sigma_{N C}}}(s) & =\Psi_{\gamma_{S_{1} D}}\left(d_{1} s\right) \times \Psi_{\gamma_{S_{2} D}}\left(d_{2} s\right) \\
& =\frac{1}{1+d_{1} \bar{\gamma}_{S_{1} D} s} \frac{1}{1+d_{2} \bar{\gamma}_{S_{2} D^{S}}} .
\end{aligned}
$$

The UPEP $\overline{\mathrm{PEP}}^{\mathrm{NC}}\left(d \mid \mathbf{W}_{d}\right)$ can be computed using the MGF method [27] as follows:

$$
\begin{aligned}
\overline{\operatorname{PEP}}^{\mathrm{NC}}\left(d \mid \mathbf{W}_{d}\right) & =\frac{1}{\pi} \int_{0}^{\pi / 2} \Psi_{\gamma_{\Sigma_{N C}}}\left(\frac{1}{\sin ^{2} \theta}\right) d \theta \\
& =\mathcal{I}_{1}\left(d_{1} \bar{\gamma}_{S_{1} D}, d_{2} \bar{\gamma}_{S_{2} D}\right),
\end{aligned}
$$

where

$$
\mathcal{I}_{1}(a, b)=\frac{1}{2}\left(1-\frac{a}{a-b} \sqrt{\frac{a}{1+a}}-\frac{b}{b-a} \sqrt{\frac{b}{1+b}}\right) .
$$

- Case 2: $d_{1}=0$. In this case, the total SNR equals $\gamma_{\Sigma_{N C}}=d_{2} \gamma_{S_{2} D}+d_{R} \gamma_{N C}$. Given the MGF of $\gamma_{N C}$ in (8), the MGF of the total SNR is given as follows:

$$
\begin{aligned}
\Psi_{\gamma_{\Sigma_{N C}}}(s) & =\Psi_{\gamma_{S_{2} D}}\left(d_{2} s\right) \times \Psi_{\gamma_{N C}}\left(d_{R} s\right) \\
& =\sum_{j=1}^{N_{r}}\left((-1)^{j-1} \sum_{\substack{n_{1}=1, \ldots, n_{j}=1 \\
n_{1} \neq \cdots \neq n_{j}}}^{N_{r}} \frac{1}{1+d_{2} \bar{\gamma}_{S_{2} D^{s}}} \frac{1}{1+d_{R} \bar{\gamma}_{N C, j} s}\right) .
\end{aligned}
$$

The UPEP $\overline{\mathrm{PEP}}^{\mathrm{NC}}\left(d \mid \mathbf{W}_{d}\right)$ is computed using the MGF method as follows:

$$
\begin{aligned}
& \overline{\operatorname{PEP}}^{\mathrm{NC}}\left(d \mid \mathbf{W}_{d}\right)=\sum_{j=1}^{N_{r}}(-1)^{j-1} \sum_{\begin{array}{r}
n_{1}=1, \ldots, n_{j}=1 \\
n_{1} \neq \cdots \neq n_{j}
\end{array}}^{N_{r}} \\
& \left.\frac{1}{\pi} \int_{0}^{\pi / 2} \frac{\sin ^{4} \theta}{\left(\sin ^{2} \theta+d_{2} \bar{\gamma}_{S_{2} D}\right)\left(\sin ^{2} \theta+d_{R} \bar{\gamma}_{N C, j}\right)} d \theta\right) \\
& =\sum_{j=1}^{N_{r}}\left((-1)^{j-1} \sum_{\substack{n_{1}=1, \ldots, n_{j}=1 \\
n_{1} \neq \cdots \neq n_{j}}}^{N_{r}} \mathcal{I}_{1}\left(d_{2} \bar{\gamma}_{S_{2} D}, d_{R} \bar{\gamma}_{N C, j}\right)\right) .
\end{aligned}
$$

- Case 3: $d_{2}=0$. Similar to Case 2 we have $\overline{\mathrm{PEP}}^{\mathrm{NC}}\left(d \mid \mathbf{W}_{d}\right)$ equals

$$
\sum_{j=1}^{N_{r}}\left((-1)^{j-1} \sum_{\substack{n_{1}=1, \ldots, n_{j}=1 \\ n_{1} \neq \cdots \neq n_{j}}}^{N_{r}} \mathcal{I}_{1}\left(d_{1} \bar{\gamma}_{S_{1} D}, d_{R} \bar{\gamma}_{N C, j}\right)\right)
$$

- Case 4: $d_{1} d_{2} d_{R} \neq 0$. In this case, $\gamma_{\Sigma_{N C}}=d_{1} \gamma_{S_{1} D}+d_{2} \gamma_{S_{2} D}+d_{R} \gamma_{N C}$. The MGF of $\gamma_{\Sigma_{N C}}$ is given as follows: 


$$
\begin{aligned}
& \Psi_{\gamma_{\Sigma_{N C}}}(s)=\Psi_{\gamma_{S_{1} D}}\left(d_{1} s\right) \times \Psi_{\gamma_{S_{2} D}}\left(d_{2} s\right) \times \Psi_{\gamma_{N C}}\left(d_{R} s\right)
\end{aligned}
$$

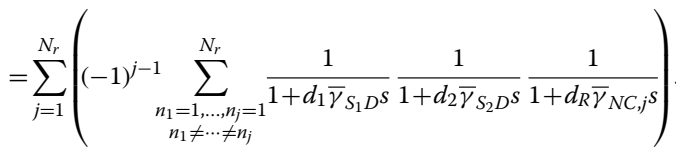

Applying the MGF method to compute the UPEP, we have:

$$
\begin{aligned}
& \overline{\operatorname{PEP}}^{\mathrm{NC}}\left(d \mid \mathbf{W}_{d}\right)=\sum_{j=1}^{N_{r}}\left(\begin{array}{c}
\sin ^{6} \theta \\
\frac{\left.\sin ^{2} \theta+d_{1} \bar{\gamma}_{S_{1} D}\right)\left(\sin ^{2} \theta+d_{2} \bar{\gamma}_{S_{2} D}\right)\left(\sin ^{2} \theta+d_{R} \bar{\gamma}_{N C, j}\right)}{n_{1} \neq \cdots \neq n_{j}=1} \int_{0} \frac{1}{\pi} \int_{0}^{\pi / 2}
\end{array}\right) \\
& =\sum_{j=1}^{N_{r}}\left(\begin{array}{c}
(-1)^{j-1} \sum_{\substack{n_{1}=1, \ldots, n_{j}=1 \\
n_{1} \neq \cdots \neq n_{j}}}^{N_{r}} \mathcal{I}_{2}\left(d_{1} \bar{\gamma}_{S_{1} D}, d_{2} \bar{\gamma}_{S_{2} D}, d_{R} \bar{\gamma}_{N C, j}\right)
\end{array}\right),
\end{aligned}
$$

where $\mathcal{I}_{2}(a, b, c)$ has been defined in Theorem 1 .

Combining these four cases gives the proof of Theorem 1 .

\section{Appendix B: proof of Theorem 2}

Let $\bar{\gamma}$ denote the referenced SNR, and let $\bar{\gamma}_{S_{1} D}=$ $G_{S_{1} D} \bar{\gamma}, \bar{\gamma}_{S_{2} D}=G_{S_{2} D} \bar{\gamma}$, and $\bar{\gamma}_{N C, j}=G_{N C, j} \bar{\gamma}$, where $G_{S_{1} D}, G_{S_{2} D}$, and $G_{N C, j}$ are constants and depend on the network topology. Note that $\bar{\gamma}$ can be one of the links, e.g., if $\bar{\gamma}$ is the average SNR of channel $S_{1} \rightarrow D$ then $G_{S_{1} D}=1$. The diversity order is defined as the negative exponent of the UPEP in log-scale when the average SNR $\bar{\gamma}$ tends to infinity

$$
\tau=-\lim _{\bar{\gamma} \rightarrow \infty}\left(\frac{\log \overline{\mathrm{PEP}}^{\mathrm{NC}}\left(d \mid \mathbf{W}_{d}\right)}{\log \bar{\gamma}}\right)
$$

Using the upper bound of UPEP [27] as $\overline{\mathrm{PEP}}^{\mathrm{NC}}\left(d \mid \mathbf{W}_{d}\right) \leq \frac{1}{2} \Psi_{\gamma_{\Sigma_{N C}}}(1 / 2)<\Psi_{\gamma_{\Sigma_{N C}}}(1 / 2)$ and recall (27), we have

$$
\tau \geq-\lim _{\bar{\gamma} \rightarrow \infty}\left(\frac{\log \Psi_{\gamma_{\Sigma_{N C}}}(1 / 2)}{\log \bar{\gamma}}\right) .
$$

We consider four cases:

- Case 1: $d_{R}=0$. In this case, all weights are located in the source-destination channels, resulting in $\Psi_{\gamma_{\Sigma_{N C}}}(1 / 2)=\Psi_{\gamma_{S_{1} D}}\left(d_{1} / 2\right) \times \Psi_{\gamma_{S_{2} D}}\left(d_{2} / 2\right)$. The diversity order in this case is given by:

$$
\begin{aligned}
\tau & \geq-\lim _{\bar{\gamma} \rightarrow \infty} \frac{\log \Psi_{\gamma_{S_{1} D}}\left(d_{1} / 2\right)}{\log \bar{\gamma}}-\lim _{\bar{\gamma} \rightarrow \infty} \frac{\log \Psi_{\gamma_{S_{2} D}}\left(d_{2} / 2\right)}{\log \bar{\gamma}} \\
& \geq-\lim _{\bar{\gamma} \rightarrow \infty}\left(\frac{\left(1+d_{1} G_{S_{1} D} \bar{\gamma} / 2\right)^{-1}}{\log \bar{\gamma}}\right)-\lim _{\bar{\gamma} \rightarrow \infty}\left(\frac{\left(1+d_{2} G_{S_{2} D} \bar{\gamma} / 2\right)^{-1}}{\log \bar{\gamma}}\right) \\
& =1+1=2 .
\end{aligned}
$$

This is enough to say the UPEP has diversity order of 2 when $d_{R}=0$, and we can write $\overline{\mathrm{PEP}}^{\mathrm{NC}}\left(d \mid d_{R}=0\right) \propto \bar{\gamma}^{-2}$.

- Case 2: $d_{1}=0$. The MGF of the total SNR in this case has a form of $\Psi_{\gamma_{\Sigma_{N C}}}(1 / 2)=\Psi_{\gamma_{S_{2} D}}\left(d_{2} / 2\right) \times \Psi_{\gamma_{N C}}\left(d_{R} / 2\right)$.

Consequently, the diversity order is given as follows:

$$
\tau \geq-\lim _{\bar{\gamma} \rightarrow \infty} \frac{\log \Psi_{\gamma_{S_{2} D}}\left(d_{2} / 2\right)}{\log \bar{\gamma}}-\underbrace{\lim _{\bar{\gamma} \rightarrow \infty} \frac{\log \Psi_{\gamma_{N C}}\left(d_{R} / 2\right)}{\log \bar{\gamma}}}_{\mathcal{J}}
$$$$
=1+\mathcal{J}
$$

where $\mathcal{J}$ is the diversity order of the best relayed signal without the direct link, which is equal to diversity order of the best relay selection for two-way relay channels. It has been shown in [6] that this diversity order is equal to $N_{r}$. Therefore, the system diversity order when $d_{1}=0$ is equal to $N_{r}+1$. In order words, $\overline{\mathrm{PEP}}^{\mathrm{NC}}\left(d \mid d_{1}=0\right) \propto \bar{\gamma}^{-\left(N_{r}+1\right)}$.

- Case 3: $d_{2}=0$. Similar to case 2, the diversity order is equal to $N_{r}+1$.

- Case 4: $d_{1} d_{2} d_{R} \neq 0$. In this case the MGF of $\gamma_{\Sigma_{N C}}$ is a product of three terms:

$$
\begin{aligned}
\Psi_{\gamma_{\Sigma_{N C}}}(1 / 2)= & \Psi_{\gamma_{S_{1} D}}\left(d_{1} / 2\right) \times \Psi_{\gamma_{S_{2} D}}\left(d_{2} / 2\right) \\
& \times \Psi_{\gamma_{N C}}\left(d_{R} / 2\right) .
\end{aligned}
$$

Substituting (31) into (28) we have

$$
\begin{aligned}
\tau \geq & -\lim _{\bar{\gamma} \rightarrow \infty} \frac{\log \Psi_{\gamma_{S_{1} D}}\left(d_{1} / 2\right)}{\log \bar{\gamma}}-\lim _{\bar{\gamma} \rightarrow \infty} \frac{\log \Psi_{\gamma_{S_{2} D}}\left(d_{2} / 2\right)}{\log \bar{\gamma}} \\
& \underbrace{-\lim _{\bar{\gamma} \rightarrow \infty} \frac{\log \Psi_{\gamma_{N C}}\left(d_{R} / 2\right)}{\log \bar{\gamma}}}_{\mathcal{\gamma} \rightarrow \infty} \\
& =1+1+\mathcal{J}=N_{r}+2 .
\end{aligned}
$$

We can write $\overline{\mathrm{PEP}}^{\mathrm{NC}}\left(d \mid d_{1} d_{2} d_{R} \neq 0\right) \propto \bar{\gamma}^{-\left(N_{r}+2\right)}$.

These four cases prove Theorem 2.

\section{Appendix C: proof of Theorem 3}

Because the relayed symbols are randomly distributed on the codeword, the weight $d_{2}$ on the relayed block can have any integer value within $[0, d]$. Denote $\mathrm{D}_{1}=\{d, 0\}$ as the 
weight pattern in which all $d$ weights are not relayed. Then the weight pattern in general has one of two forms, $\mathrm{D}_{1}=$ $\{d, 0\}$ and $\mathbf{D}_{d} \neq \mathrm{D}_{1}$. Using the MGF method, the UPEP can be computed as follows:

$$
\overline{\operatorname{PEP}}\left(d \mid \mathbf{D}_{d}\right)=\frac{1}{\pi} \int_{0}^{\pi / 2} \Psi_{\gamma_{\Sigma}}\left(\frac{1}{\sin ^{2} \theta}\right) d \theta .
$$

- Case 1: $\mathbf{D}_{d}=D_{1}$. In this case, all $d$ weights are located in the source-destination block, resulting in $\gamma_{\Sigma}=d \gamma_{S D}$ and $\Psi_{\gamma_{\Sigma}}(s)=\Psi_{\gamma_{S D}}(d s)$. In this case, we have

$$
\begin{aligned}
\overline{\operatorname{PEP}}\left(d \mid \mathrm{D}_{1}\right) & =\frac{1}{\pi} \int_{0}^{\pi / 2} \frac{\sin ^{2} \theta}{\sin ^{2} \theta+d \bar{\gamma}_{S D}} d \theta \\
& =\frac{1}{2}\left(1-\sqrt{\frac{d \bar{\gamma}_{S D}}{1+d \bar{\gamma}_{S D}}}\right) .
\end{aligned}
$$

- Case 2: $\mathbf{D}_{d} \neq \mathrm{D}_{1}$. There is always $d_{2}$ weights are relayed, resulting in $\Psi_{\gamma_{\Sigma}}(s)=\Psi_{\gamma_{S D}}(d s) \times \Psi_{\gamma_{R^{\star}}}\left(d_{2} s\right)$. From (9) we have

$$
\begin{aligned}
& \overline{\operatorname{PEP}}\left(d \mid \mathbf{D}_{d}\right)=\sum_{j=1}^{N_{r}}\left((-1)^{j-1} \sum_{\substack{n_{1}=1, \ldots, n_{j}=1 \\
n_{1} \neq \cdots \neq n_{j}}}^{N_{r}} \frac{1}{\pi} \int_{0}^{\pi / 2} \frac{\sin ^{4} \theta}{\left(\sin ^{2} \theta+d \bar{\gamma}_{S D}\right)\left(\sin ^{2} \theta+d_{2} \bar{\gamma}_{R^{\star}, j}\right)} d \theta\right) \\
& =\sum_{j=1}^{N_{r}}\left((-1)^{j-1} \sum_{\substack{n_{1}=1, \ldots, n_{j}=1 \\
n_{1} \neq \cdots \neq n_{j}}}^{N_{r}} \mathcal{I}_{1}\left(d \bar{\gamma}_{S D}, d_{2} \bar{\gamma}_{R_{i}^{*}, j}\right)\right),
\end{aligned}
$$

where $\mathcal{I}_{1}(a, b)$ is defined in Theorem 1 , and $\bar{\gamma}_{R^{\star}, j}$ is defined in Section 3.2.

\section{Appendix D: proof of Theorem 4}

Let $\bar{\gamma}$ denote the referenced SNR, and $\bar{\gamma}_{S D}=G_{S D} \bar{\gamma}, \bar{\gamma}_{R^{\star}}=$ $G_{R} \bar{\gamma}$, where $G_{S D}$ and $G_{R}$ are constants depending on the network topology. We employ the upper bound of UPEP to derive diversity order for $\overline{\operatorname{PEP}}\left(d \mid \mathbf{D}_{d}\right)$ as [27]

$$
\tau \geq-\lim _{\bar{\gamma} \rightarrow \infty}\left(\frac{\log \Psi_{\gamma_{\Sigma}}(1 / 2)}{\log \bar{\gamma}}\right)
$$

We consider two cases.

- Case 1: $\mathbf{D}_{d}=\mathrm{D}_{1}=\{d, 0\}$. There is no symbol helped by the relay and thus $\Psi_{\gamma_{\Sigma}}(1 / 2)=\Psi_{\gamma_{S D}}(d / 2)$. The diversity order in this case is given as

$$
\begin{aligned}
\tau & \geq-\lim _{\bar{\gamma} \rightarrow \infty} \frac{\log \Psi_{\gamma_{S D}}(d / 2)}{\log \bar{\gamma}} \\
& \geq-\lim _{\bar{\gamma} \rightarrow \infty}\left(\frac{\left(1+d G_{S D} \bar{\gamma} / 2\right)^{-1}}{\log \bar{\gamma}}\right)=1,
\end{aligned}
$$

which states that the UPEP has diversity order of one when $d_{2}=0$. We may write $\overline{\operatorname{PEP}}\left(d \mid \mathrm{D}_{1}\right) \propto \bar{\gamma}^{-1}$.
- Case 2: $\mathbf{D}_{d} \neq \mathrm{D}_{1}$, then $\Psi_{\gamma_{\Sigma}}(1 / 2)=\Psi_{\gamma_{S D}}(d / 2) \times \Psi_{\gamma_{R^{\star}}}\left(d_{2} / 2\right)$. Consequently, the diversity order is given as

$$
\begin{aligned}
\tau & \geq-\lim _{\gamma \rightarrow \infty} \frac{\log \Psi_{\gamma_{S D}}(d / 2)}{\log \bar{\gamma}} \underbrace{-\lim _{\gamma \rightarrow \infty} \frac{\log \Psi_{\gamma_{R^{\star}}}\left(d_{2} / 2\right)}{\log \bar{\gamma}}}_{\tau_{\text {Sel }}} \\
& =1+\tau_{\text {Sel }},
\end{aligned}
$$

where $\tau_{S e l}$ is the diversity order of the best relay signal (without the direct link). It is shown in [3] that the best relay selection achieves full diversity order of $N_{r}$. Therefore, we can write $\overline{\mathrm{PEP}}\left(d \mid \mathbf{D}_{d} \neq \mathrm{D}_{1}\right) \propto \bar{\gamma}^{-\left(N_{r}+1\right)}$.

Combining these two cases, we complete the proof of Theorem 4. $[9,17,18]$.

\section{Acknowledgements}

The research work is supported in part by the Luxembourg FNR Core 2013 SeMiGod under the grant code I2R-SIG-PFN-13SEMI.

\section{Authors' contributions}

TXV substantially contributes to this paper. PD, SC, and BO equally contribute to the manuscript. All authors read and approved the final manuscript.

\section{Competing interests}

The authors declare that they have no competing interests.

\section{Publisher's Note}

Springer Nature remains neutral with regard to jurisdictional claims in published maps and institutional affiliations.

\section{Author details}

${ }^{1}$ Interdisciplinary Centre for Security, Reliability and Trust (SnT), University of Luxembourg, Luxembourg, Luxembourg. ${ }^{2}$ Laboratory of Signals and Systems (LSS), French National Center for Scientific Research (CNRS) - CentraleSupélec - University of Paris-Sud 11, Paris, France.

Received: 15 December 2016 Accepted: 18 April 2017

Published online: 02 May 2017

\section{References}

1. A Sendonaris, E Erkip, B Aazhang, User cooperation diversity - part I: system description. IEEE Trans. Commun. 51(11), 1927-1938 (2003)

2. EV Meulen, Three-terminal communication channels. Adv. Appl. Probab. 3, 120-154 (1971)

3. A Bletsas, A Khisti, DP Reed, A Lippman, A Simple cooperative diversity method based on network path selection. IEEE J. Sel. Areas Commun. 24(3), 659-672 (2006)

4. L Sungeun, H Myeongsu, H Daesik, in Vehicular Technology Conference, 2009. VTC Spring 2009. IEEE 69th. Average SNR and Ergodic Capacity Analysis for Proactive and Reactive DF Relaying over Rayleigh Fading Channels, (Barcelona, 2009), pp. 1-5

5. R Ahlswede, C Ning, SYR Li, RW Yeung, Network information flow. IEEE Trans. Inf. Theory. 46(4), 1204-1216 (2000)

6. Y Li, RHY Louie, B Vucetic, Relay selection with network coding in Two-Way Relay Channels. IEEE Trans. Veh. Technol. 59(9), 4489-4499 (2010)

7. $Y$ Jing, $\mathrm{H}$ Jafarkhani, Single and multiple relay selection schemes and their achievable diversity orders. IEEE Trans. Wirel. Commun. 8(3), 1414-1423 (2009)

8. S Atapattu, Y Jing, H Jiang, C Tellambura, Relay selection schemes and performance analysis approximations for two-way networks. IEEE Trans. Commun. 61(3), 987-998 (2013) 
9. MD Renzo, On the achievable diversity of repetition-based and relay selection network-coded cooperation. IEEE Trans. Commun. 62(7), 2296-2313 (2014)

10. C Peng, Q Zhang, M Zhao, Y Yao, W Jia, On the performance analysis of network-coded cooperation in wireless networks. IEEE Trans. Wirel. Commun. 7(8), 3090-3097 (2008)

11. H Topakkaya, W Zhengdao, Wireless network code design and performance analysis using diversity-multiplexing tradeoff. IEEE Trans. Commun. 59(2), 488-496 (2011)

12. TX Vu, P Duhamel, M Direnzo, On the diversity of network-coded cooperation with decode-and-forward relay selection. IEEE Trans. Wirel. Commun. 14(8), 4369-4378 (2015)

13. Z Ding, T Ratnarajah, K Leung, On the study of network coded AF transmission protocol for wireless multiple access channels. IEEE Trans. Wirel. Commun. 7(11), 4568-4574 (2008)

14. W Geng, $T L v$, in Wireless personal multimedia communications (WPMC) 2012 15th International Symposium on. An opportunistic network coding cooperative scheme for wireless uplink multi-relay cooperative networks, (Taipei, 2012), pp. 148-152

15. W Guan, K Liu, Diversity analysis of analog network coding with multi-user interferences. IEEE Trans. Wirel. Commun. 12(2), 668-679 (2013)

16. SS Ikki, MH Ahmed, Performance of multiple-relay cooperative diversity systems with best relay selection over Rayleigh fading channels. EURASIP J. Adv. Signal Process. 2008, 580368 (2008)

17. W Jiang, T Kaiser, AJH Vinck, A robust opportunistic relaying strategy for co-operative wireless communications. IEEE Trans. Wirel. Commun. 15(4), 2642-2655 (2016)

18. S Yadav, PK Upadhyay, Impact of outyeard channel estimates on opportunistic two-way ANC-based relaying with three-phase transmissions. IEEE Trans. Veh. Technol. 64(12), 5750-5766 (2015)

19. T Wang, A Cano, GB Giannakis, JN Laneman, High-performance cooperative demodulation with decode-and-forward relays. IEEE Trans. Commun. 55(7), 1427-1438 (2007)

20. A Nasri, R Schober, M Uysal, Performance and optimization of network-coded cooperative diversity systems. IEEE Trans. Commun. PP(99), 1-12 (2013)

21. XT Vu, M DiRenzo, P Duhamel, in Personal, indoor and mobile radio communications, 2013. PIMRC'13., 24th IEEE International Symposium on. BER Analysis of Joint Network/Channel decoding in Block Rayleigh fading channels, (London, 2013), pp. 698-702

22. A Eckford, J Chu, R Adve, Low complexity and fractional coded cooperation for wireless networks. IEEE Trans. Wirel. Commun. 7(5), 1917-1929 (2008)

23. M Khormuji, E Larsson, Cooperative transmission based on decode-and-forward relaying with partial repetition coding. IEEE Trans. Wirel. Commun. 8(4), 1716-1725 (2009)

24. T Cover, A Gamal: Capacity theorems for the relay channel. IEEE Trans. Inf. Theory. 25(5), 572-584 (1979)

25. XT Vu, M DiRenzo, P Duhamel, Multiple Access Relaying with Network Coding: Iterative Network/Channel Decoding with Imperfect CSII EURASIP. J. Adv. Signal Process. 1(170) (2013)

26. L Bahl, J Cocke, F Jelinek, J Raviv, Optimal. IEEE Trans. Inf. Theory. 20(2), 284-287 (1974)

27. MK Simon, MS Alouini, Digital communication over fading channels Wiley series in telecommunications and signal processing, 2nd edition. (Hoboken, Wiley, 2005)

28. A Glavieux, Channel coding in communication networks: from theory to turbo codes. (Wiley \& Sons Ltd, 2007)

29. TX Vu, NQB Vo, M Di Renzo, P Duhamel, in Signal processing advances in wireless communications (SPAWC), 2013 IEEE 14th International Workshop on. Performance Analysis of Relay Networks with Channel code in Low SNR Regime, (Darmstadt, 2013), pp. 1948-3244

30. R Narasimhan, Finite-SNR diversity-multiplexing tradeoff for correlated Rayleigh and Rician MIMO channels. IEEE Trans. Inf. Theory. 52(9), 3965-3979 (2006)

31. P Frenger, P Orten, T Ottosson, Convolutional codes with optimum distance spectrum. IEEE Commun. Lett. 3(11), 317-319 (1999)

32. L Zhang, D Tse, Diversity and multiplexing: a fundamental tradeoff in multiple-antenna channels. IEEE Trans. Inf. Theory. 52(9), 3965-3979 (2006)

\section{Submit your manuscript to a SpringerOpen ${ }^{\circ}$ journal and benefit from:}

- Convenient online submission

Rigorous peer review

- Immediate publication on acceptance

- Open access: articles freely available online

- High visibility within the field

- Retaining the copyright to your article

Submit your next manuscript at $\gg$ springeropen.com 